\title{
Nonequilibrium Quantum Breakdown in a Strongly Correlated Electron System
}

\author{
Takashi Oka, Hideo Aoki \\ Department of Physics, University of Tokyo, Hongo, Tokyo 113-0033, Japan \\ oka@cms.phys.s.u-tokyo.ac.jp, aoki@cms.phys.s.u-tokyo.ac.jp
}

\section{Introduction}

During the past decades, there has been an increasing fascination and surprises with diverse quantum many-body effects. With the magical touch of interaction a simple electron system may assume insulating, metallic, magnetic or superconducting states according as the control parameters are changed. Strongly correlated electron systems, as exemplified by the high-Tc superconductors and their host materials realized in transition-metal oxides, as well as by organic metals, have provided us with an ideal playground, where various crystal structures with band-filling control and band-width control etc provide the richness in the phase diagram [39].

On the other hand, there is a long history of the interests in non-equilibrium phase transitions. Statistical mechanically, there is an intriguing problem of how we can generally define the notion of a "phase" in non-equilibrium systems, but we can still discuss individual systems in specified non-equilibrium conditions to extract more general viewpoints. Now, if we combine the above two ingredients, namely, if we consider strongly-correlated electron systems in non-equilibrium, we plunge into an even more fascinating physics. In fact, recent years have witnessed an upsurge of interests in non-equilibrium states in many-body systems with drastic changes in the electronic states in strong dc electric fields, in intense laser fields, etc.

Developments in fabrication techniques such as realization of clean thin films with electrodes attached have triggered several groundbreaking experiments, e.g., non-linear transport measurements in thin films [1, 60, 19, 50, 2, 4, in layered systems 24 and observations of clean metallic states in heterostructures [3]. Non-linear phenomena in correlated electron systems now begin to attract interests in a wide range of researchers: One obvious area of application is future-generation electronic devices, where a high sensitivity of a

to be published in "Quantum and Semi-classical Percolation \& Breakdown",

Lecture Notes in Physics (LNP), Springer-Verlag 


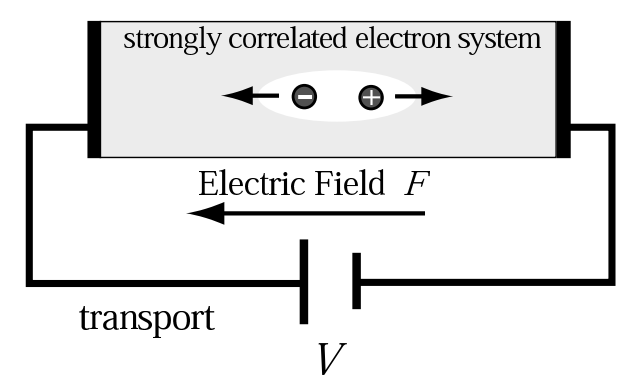

Fig. 1. Non-linear transport and optical response

system near a phase boundary to external conditions may lead to drastic functionalities [1. However, even more attractive is its relevance to fundamental physics, especially, to non-equilibrium statistical physics, where we can observe the behavior of various phase transitions taking place under nonequilibrium conditions.

The purpose of the present article is to discuss the nonequilibrium metalinsulator transition in strongly correlated electron systems [57, 58, 7, 8, which is known, for equilibrium systems, as Mott's transition. Before going into detail, we first give a brief introduction of the transition, and discuss how quantum breakdown through non-adiabatic transitions in nonequilibrium becomes relevant in non-linear transports.

The model we study is the single-band Hubbard model, which is the simplest possible one that captures many essential properties of correlated electron physics. The Hamiltonian reads

$$
H_{0}=-t_{\mathrm{hop}} \sum_{\langle i, j\rangle \sigma}\left(c_{i \sigma}^{\dagger} c_{j \sigma}+\text { h.c. }\right)+U \sum_{i} n_{i \uparrow} n_{i \downarrow},
$$

where $c_{i \sigma}$ annihilates an electron on site $i$ with spin $\sigma, n_{i \sigma}=c_{i \sigma}^{\dagger} c_{i \sigma}$ the number operator, $U$ the strength of the on-site Coulomb repulsion, and $t_{\text {hop }}$ the hopping integral. The filling $n=\frac{1}{L} \sum_{i=1}^{L}\left\langle n_{i \uparrow}+n_{i \downarrow}\right\rangle$, with $L$ the number of sites, is an important parameter, which changes the groundstate property drastically.

When the band is half-filled with one electron per site on average $(n=1)$, each electron tends to be localized on a separate lattice site and the spin tends to be antiferromagnetically correlated. When $U / t$ is large enough, the groundstate is insulating, which is called Mott's insulator (Fig2(a)), and the groundstate is separated from the charge excited states with a many-body energy gap - Mott gap. When we inject carriers (usually with a chemical doping by adding or replacing to other elements) to increase (electron doping) or decrease (hole doping) the filling from unity, the Mott gap collapses for large enough doping, and the system becomes metallic. This is the 


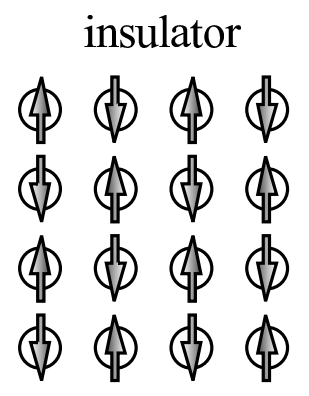

(a)

\section{doped metal (equilibrium)}

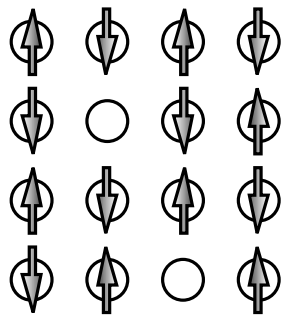

(b)

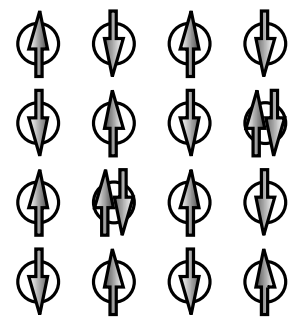

(c)

Fig. 2. Metal-insulator transition in equilibrium due to doping: (a) A Mott insulator realized at half filling. (b) A hole-doped metallic state. (c) An electron-doped metallic state.

metal-insulator transition or the Mott transition, which is widely observed in strongly correlated materials. In these materials, a state occupied simultaneously by up an down-spin electrons - which we call a doublon - and holes carry the current. After the discovery of the high-temperature superconductivity in cuprates, carrier-doped Mott insulators have been subject of a huge number of experimental and theoretical studies.

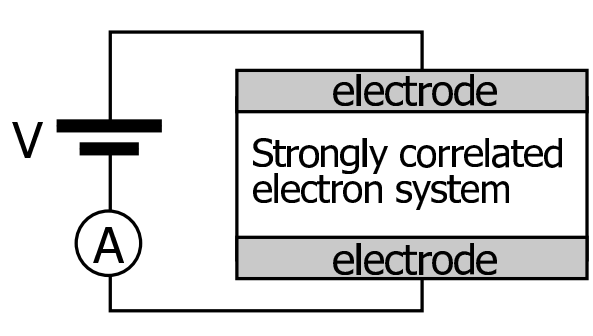

(a)

\section{non-linear transport}

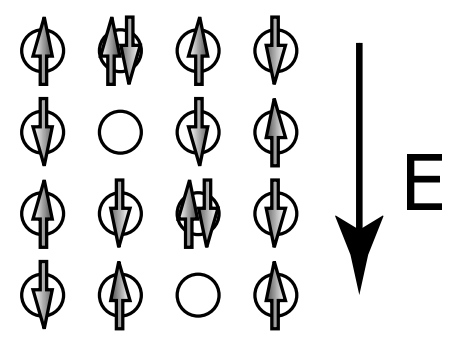

(b)

Fig. 3. (a) Schematic experimental configuration. (b) Carriers (doublons and holes) created by an external electric field.

Now, let us consider what will happen if we attach a set of electrodes to a strongly-correlated sample, and apply a large bias voltage across the electrodes (Fig. 3 (a)). Although the setup may seem simple enough, there is a profound physics involved.

In regions near the electrodes (or near the interface in the case of heterojunctions between a strongly-correlated and ordinary materials), a "band bending" similar to doped semiconductors can take place and lead to an in- 
terface Mott transition when the filling becomes one 59. The width of the insulating layer changes as the applied bias is changed, which dominates the behavior of the non-linear transport (i.e., the $I-V$ characteristics). The result (with DMRG + Hartree potential) for the band-bending effects in this case can be understood if we assume a local equilibrium for the relation between the density of electrons and the potential. The local properties are determined by the Hartree potential governed by Poisson's equation, which in turn determines the local chemical potential and controls the metal-insulator transition.

Even more interesting, however, is the case where we no longer have local equilibrium. Specifically, a quantum many-body breakdown of a Mott's insulator takes place when the applied electric field is large enough and creates doublons and holes in the Mott insulating groundstate (Fig. 3 (b)) [58, 8, 57.

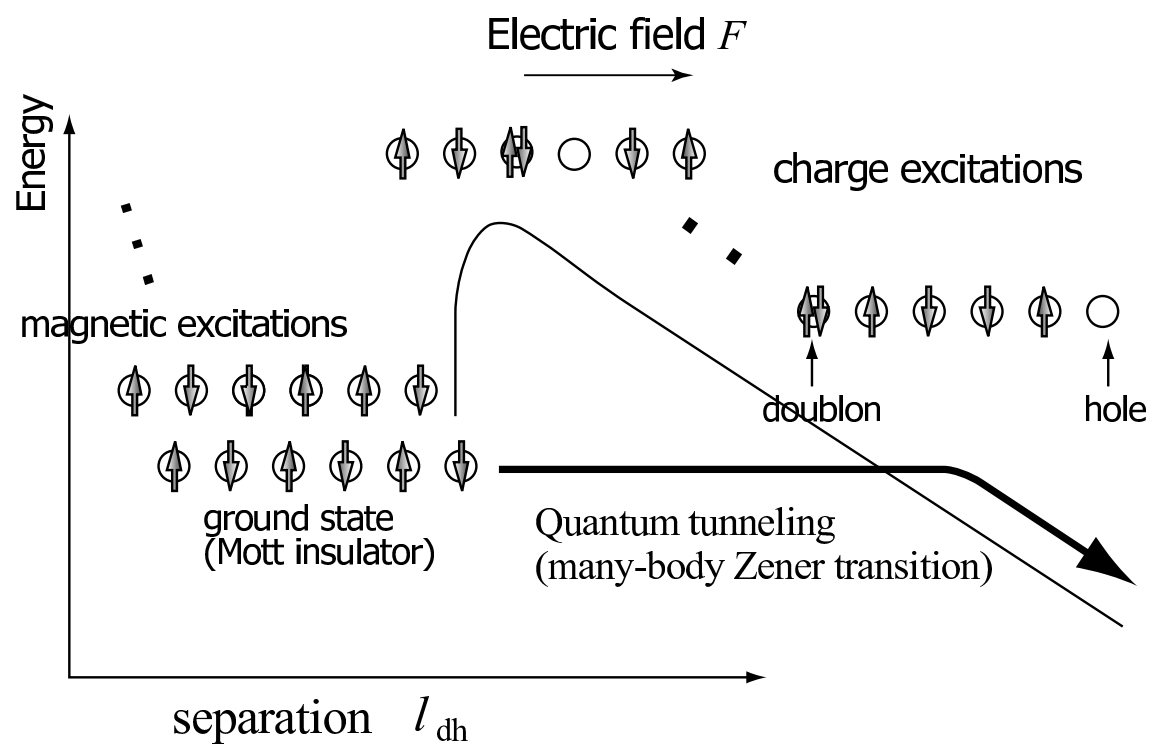

Fig. 4. Dielectric breakdown of a Mott insulator in a strong electric field due to many-body Landau-Zener transition: The groundstate and excited states with charge excitations are separated by an energy barrier, and quantum tunneling among many-body states takes place when the electric field is strong enough.

The creation mechanism is a many-body analog of the "Zener breakdown", well-known in semiconductor physics 68. Namely, while we cannot use the notion of the electronic band structure for correlated electron systems, we can envisage the carrier-creation process as a tunneling across a kind of barrier. As displayed in Fig. 4, production of carriers occurs through tunneling between the Mott insulating groundstate and excited states with doublons and holes. If we denote the distance between a doublon and a hole by $l_{\mathrm{dh}}$, the energy profile as a function of $l_{\mathrm{dh}}$ roughly reads 


$$
\Delta E \sim U-l_{\mathrm{dh}} F,
$$

where $F$ is the strength of the electric field. The profile curve reaches the energy before the creation of the doublon-hole pair for the separation at which

$$
\Delta E \sim U-\bar{l}_{\mathrm{dh}} F=0,
$$

to which the tunneling becomes possible. There is a threshold field strength for this process to occur. This is because larger quantum fluctuations are required to have a larger separation between doublon and hole in the Mott insulator.

In other words, the overlaps of many-body wave function of the groundstate and excited states decrease rapidly for large $l_{\mathrm{dh}}$. We can formulate this with the Landau-Zener picture in the time-dependent gauge for the external electric field, for which, as we shall show below [57](eq.(51)), the threshold field strength is given by

$$
F_{\text {th }}=\frac{\Delta_{\mathrm{c}}(U)^{2}}{8 t_{\mathrm{hop}}}
$$

where $\Delta_{\mathrm{c}}(U)$ is the charge gap (i.e., the Mott gap), and the tunneling rate per length is given by

$$
\Gamma(F) / L=-\frac{2 F}{h} a \ln [1-p(F)],
$$

where $p(F)=e^{-\pi \frac{F_{\text {th }}}{F}}$ is the tunneling probability and $a$ a non-universal constant depending on the detail of the system. The tunneling rate $\Gamma(F) / L$, being related to the production rate of carriers, is directly related to physical properties in the bulk if the interface effect is neglected. Indeed, such a nonlinearity in the $I-V$ characteristics has been observed in real materials, most prominently in a one-dimensional copper oxide [41].

Another interesting consequence of eq.(3) is that it gives the "critical separation" of the doublon-hole excitation $\bar{l}_{\mathrm{dh}}=U / F_{\mathrm{th}}$. This has to do with the convex shape of the energy profile against $l_{\mathrm{dh}}$ (Fig 4), which is reminiscent of the energy profile for the standard nucleation theory that treats the critical size of a stable-phase droplet to grow without being crushed, although the physics involved is quite different. In the present case, when the field is greater than the threshold, the electric-field induced metallic state, where doublon-hole pairs continue to be created, becomes the stable phase.

The first goal of this article is to derive the relations presented above and study the creation mechanism of carriers (this part is the extended argument of our papers [58, 57]). We need to treat the process quantum mechanically and in a many-body formulation. In doing so, we present a renewed and unified interpretation of the Zener transition of insulators. The key quantity is the effective Lagrangian of quantum dynamics (see $\$ 2.2$ for a detailed introduction) which is define by [57.

$$
\mathcal{L}(F)=-\frac{i}{L^{d}} \lim _{t \rightarrow \infty} \frac{1}{t} \ln \Xi(t),
$$


where $\Xi(t)$ is the groundstate-to-groundstate transition amplitude and $L^{d}$ is the volume of the $d$ dimensional system and $L$ the linear size. There is a deep relation between the theories of dielectric breakdown in condensed matter and a branch in quantum field theory known as non-linear quantum electrodynamics (QED) (table: 1). The effective Lagrangian defined above coincides with the Heisenberg-Euler effective Lagrangian for non-adiabatic evolution [20, 57. The effective Lagrangian have been used to study the Schwinger mechanism of electron-positron pair production from the QED vacuum in strong electric fields [52]. In fact, we show that the Schwinger mechanism and the Zener tunneling are equivalent, where the effective action coincides if we consider the breakdown of simple Dirac type band insulators. Furthermore, the effective action gives the non-adiabatic extension of the Berry phase theory of polarization.

\begin{tabular}{l|c|c}
\hline & dielectric breakdown in cond. matter & non-linear QED \\
\hline \hline mechanism & Zener breakdown [58] & Schwinger mechanism [52] \\
excitation & electron (doublon)-hole pair & electron-positron pair \\
effect of interaction & many-body Landau-Zener & back reaction \\
non-linear polarization & cross correlation (ME effect) & photon-photon interaction \\
& non-adiabatic Berry phase theory [57 & - \\
\hline
\end{tabular}

Table 1. Relation between the theory of dielectric breakdown in condensed matter and non-linear QED from the point of view of the effective Lagrangian.

In the latter part of the article we shall discuss the effect of annihilation of doublon-hole pairs (Fig. (5). In a one-dimensional system, a doublon and a hole cannot pass each other without being pair-annihilated even as virtual processes. Since the groundstate is locally stable, the many-body state tends to remain in the ground state, but there should be a finite probability for the state to "branch into" excited states through many paths in the manybody energy space. Thus, the long-time behavior of the wave function involves numerous scattering processes in the energy space, where, as we shall see, the phase interference plays a key role. We can indeed regard the phase before the dielectric breakdown takes place as a dynamical localization in the many-body energy space, which reduces the tunneling rate and makes the groundstate survive [8, 7. A statistical mechanical treatment helps in understanding this, and we briefly discuss it in terms of the quantum walk.

A brief comment on the numerical methods used in the article: In order to understand the non-equilibrium processes, we need to integrate the timedependent, many-body Schrödinger equation to look at the evolution of the many-body wave function in, say, the Hubbard model in strong electric fields. This is a formidable task, for which no analytically exact treatment is known, so that we rely on several numerical methods, which include the exact diagonalization and the time-dependent density matrix renormalization group 


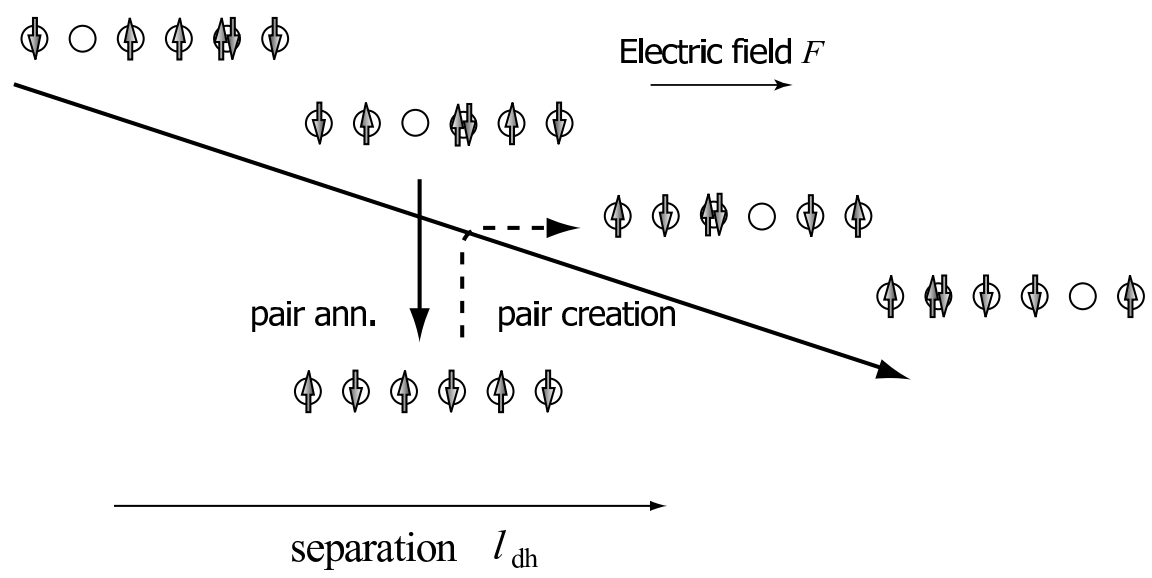

Fig. 5. Annihilation processes for carriers in a correlated electron system.

method (td-DMRG; The version of td-DMRG we adopt is the one proposed by White and Feiguin [63]).

\section{Non-adiabatic evolution and pair creation of carriers}

\subsection{Electric fields and gauge transformation}

When we describe a system in finite electric fields, we can choose from two gauges. One is the case where we have a slanted electrostatic potential, with the gauge field $A^{\mu}=(F x, 0)$ for a one-dimensional system with $F=e E$ being the electric field, while the other represents the electric field via a timedependent vector potential, $A^{\mu}=(0,-F t)$.

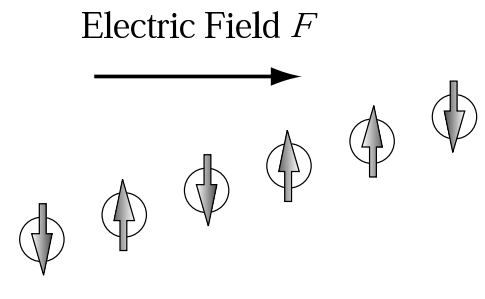

(a)

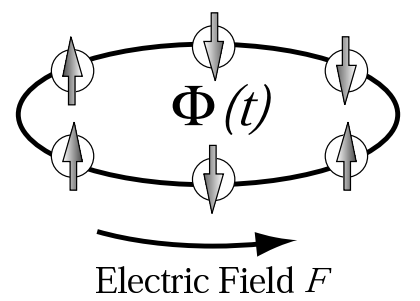

(b)

Fig. 6. (a) Time-independent gauge. (b) Time-dependent gauge.

In the first gauge, the tilted potential enters in the Hamiltonian as

$$
H(F)=H_{0}+F \hat{X}, \quad \hat{X}=\sum_{j} j n_{j},
$$


where $\hat{X}$ is the position operator 48 and $H_{0}$ the original Hamiltonian. This gauge is incompatible with systems with periodic boundary conditions. The Hamiltonian $H(F)$ in fact becomes an unbounded operator in an infinite system, since one can lower the energy indefinitely by moving an electron to $j \rightarrow-\infty$.

In the other gauge, which we call the time-dependent gauge, the hopping term of the Hamiltonian becomes time-dependent as

$$
H(\phi(t))=-t_{\text {hop }} \sum_{i \sigma}\left(e^{i \frac{2 \pi}{L} \phi(t)} c_{i+1 \sigma}^{\dagger} c_{i \sigma}+\text { h.c }\right)+\hat{V},
$$

where $\Phi(t)$ represents a time-dependent Aharonov-Bohm(AB) flux,

$$
\phi(t) \equiv \Phi(t) / \Phi_{0}=F L t / h .
$$

Physically, this gauge amounts to considering a periodic system (a ring) and a magnetic flux piercing the ring, where the time-dependent flux induces electric fields by Faraday's law (Fig. [6). For a higher-dimensional system, the ring becomes a (generalized) torus. The time-dependent gauge is suited for periodic systems since it is compatible with the lattice translation symmetry. The electric current operator is obtained by differentiating the Hamiltonian by $A^{1}$ as

$$
J(\phi)=-\frac{d H(\phi)}{d A^{1}}=-i t_{\mathrm{hop}} \sum_{i \sigma}\left(e^{i \frac{2 \pi}{L} \phi} c_{i+1 \sigma}^{\dagger} c_{i \sigma}-e^{-i \frac{2 \pi}{L} \phi} c_{-i \sigma}^{\dagger} c_{i+1 \sigma}\right) .
$$

There exists an important operator relation among $H, J$ and $\hat{X}$,

$$
J(\phi)=\frac{i}{\hbar}[H(\phi), \hat{X}],
$$

which comes from Heisenberg's equation of motion for the current operator, $J(\phi)=\frac{d}{d t} \hat{X}$.

We can relate the two gauges with a twist operator [9] defined by

$$
g(\phi)=e^{-i \frac{2 \pi}{L} \phi \hat{X}},
$$

and the two Hamiltonians are related by a gauge transformation generated by the twist operator, i.e.,

$$
H(F)=g^{\dagger}(\phi(t)) H(\phi(t)) g(\phi(t))-i g^{\dagger}(\phi(t)) \partial_{t} g(\phi(t)) .
$$

\subsection{Heisenberg-Euler effective Lagrangian}

We first discuss the non-adiabatic evolution of electron wave functions in insulators (either one-body or many-body) in strong electric fields. Let us consider an insulator at $T=0$ and $F=0$, which is described by the groundstate wave 
function $\left|\Psi_{0}\right\rangle$. We then switch on the electric fields at $t=0$ to study the quantum mechanical evolution of the system. We limit our discussions to coherent dynamics and ignore the effect of dissipation due to heat bath degrees of freedom as well as boundary effects near the electrodes.

A key quantity to study the non-adiabatic evolution and quantum tunneling in strong electric fields is the (condensed-matter counterpart to the) effective Lagrangian introduced for QED by Heisenberg and Euler 20. In the time-independent gauge, the electrons are described by the solution of the Schrödinger equation,

$$
|\Psi(t)\rangle=e^{-i t H(F)}\left|\Psi_{0}\right\rangle,
$$

where we have put $\hbar=1$. The overlap of the solution with the groundstate for $F=0$ - groundstate-to-groundstate transition amplitude — should contain the information on the tunneling processes, so we define

$$
\Xi(t)=\left\langle\Psi_{0}\left|e^{-i t H(F)}\right| \Psi_{0}\right\rangle e^{i t E_{0}},
$$

where we have factored out the trivial dynamical phase of the groundstate, $E_{0}=\langle\Psi|H(F=0)| \Psi\rangle$. In the case of the time-dependent gauge, we need to be careful, since the groundstate is $\phi$ dependent. If we denote $|0 ; \phi\rangle$ as the instantaneous groundstate of $H(\phi)$, the groundstate-to-groundstate transition amplitude becomes

$$
\Xi(\tau)=\left\langle 0 ; \phi(\tau)\left|\hat{T} e^{-\frac{i}{\hbar} \int_{0}^{\tau} H(\phi(s)) d s}\right| 0 ; \phi(0)\right\rangle e^{\frac{i}{\hbar} \int_{0}^{\tau} E_{0}(\phi(s)) d s},
$$

where $\hat{T}$ stands for the time ordering, and $E_{0}(\phi)=\langle 0 ; \phi(\tau)|H(\phi)| 0 ; \phi\rangle$ the dynamical phase of the groundstate.

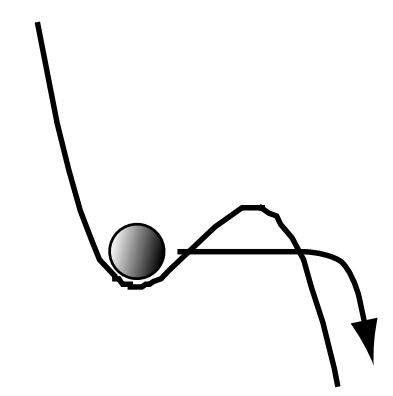

Fig. 7. The original problem studied by Callan and Coleman in which quantum tunneling from an unstable vacuum is considered 23.

We define [57] the effective Lagrangian by

$$
\mathcal{L}(F)=-\frac{i}{L^{d}} \lim _{t \rightarrow \infty} \frac{1}{t} \ln \Xi(t),
$$


where $L^{d}$ is the volume of the $d$ dimensional system with a linear dimension of $L$. We can also regard the Lagrangian as the exponent of the asymptotic behavior of the amplitude, $\Xi(\tau) \sim e^{i \tau L^{d} \mathcal{L}(F)}$. When the electric field is large enough, the groundstate becomes unstable with the quantum tunneling to excited states activated. The tunneling rate is described by the imaginary part of the effective Lagrangian,

$$
\Gamma(F) / L^{d} \equiv 2 \operatorname{Im} \mathcal{L}(F),
$$

which gives the rate of the exponential decay of the vacuum (groundstate). In the quantum field theory, the decay rate of an unstable vacuum has been discussed by Callan and Coleman, where the tunneling takes place when the potential is suddenly changed by an external field 23] (Fig. 17).

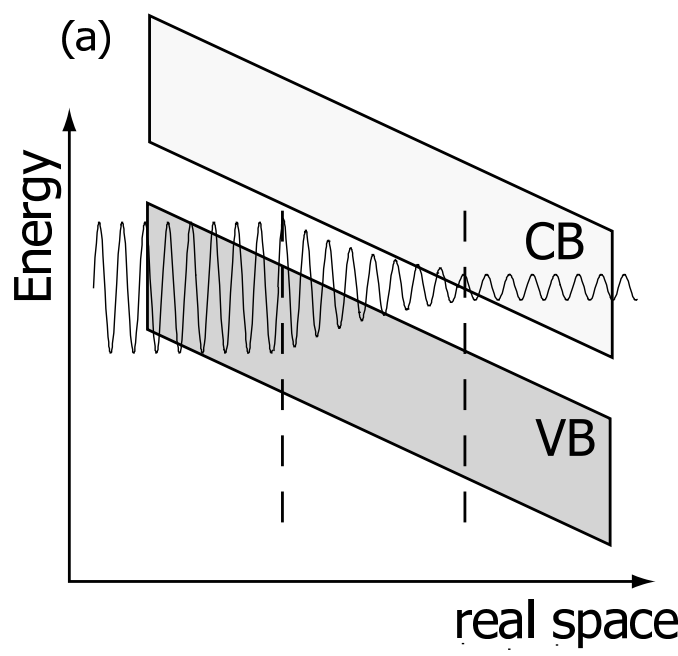

(b)

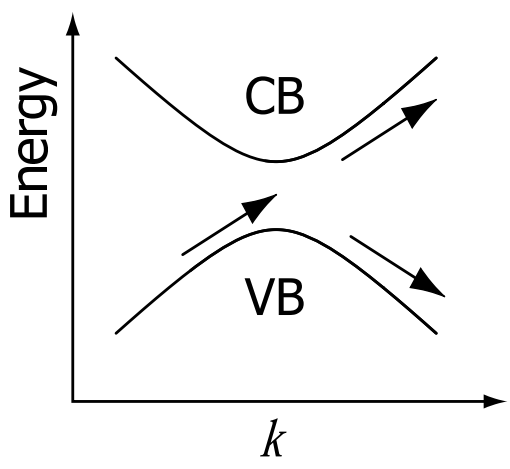

Fig. 8. Two models of the dielectric breakdown studied by Zener. (a) Timeindependent gauge. (b) Time-dependent gauge.

As we shall see later in several models, in the theory of dielectric breakdown, the tunneling corresponds to creation of charge carriers. In band insulators the carriers are electrons and holes, while in Mott insulators they are doublons and holes. If we neglect boundary effects and assume that all the carriers are absorbed by electrodes, we can conclude that the tunneling rate is proportional to the leakage current, i.e.,

$$
J_{\text {leak }} \propto \Gamma(F) / L^{d} .
$$

Indeed, this is the original picture of Zener when he calculated the leakage current in a simple band insulator 8 Zener has studied the dielectric breakdown in a simple one-dimensional insulator using the time-independent gauge 
69 as well as the time-dependent gauge 68. In the former, he has calculated the tunneling probability of Bloch functions in constant electric fields to obtain the tunneling rate. In the time-dependent gauge, he has described the problem as a system with a time-dependent Hamiltonian represented by a two-by-two matrix to study the tunneling near the level anti-crossing, which is now known as the Landau-Zener transition [38, 68.

The reason why we have called $\mathcal{L}(F)$ the effective Lagrangian is that it coincides with the Heisenberg-Euler effective Lagrangian in QED [20. They have studied the dynamics and nonlinear responses of the QED vacuum in strong electric fields by calculating the effective Lagrangian (for a review, see e.g. [15]). By integrating out high-energy degrees of freedom (polarization processes due to electron-positron creation/annihilation) they arrived at an effective description of the low-energy degrees of freedom, namely the quantum correction, originating from the fluctuation of the QED vacuum, to the Maxwell theory of electromagnetism. Indeed, if we apply our formalism to band insulators with Dirac-type (mass-gapped) dispersions, the effective Lagrangian coincides with the Heisenberg-Euler Lagrangian with some modifications coming from the Brillouin zone structure of the Bloch waves as will be shown in the next section. The correspondence between the two phenomena is straightforward: The ground-state of the insulator translates to the QED vacuum, charge excitations to the electron-positron pairs. The tunneling rate also has its QED counterpart, namely the vacuum decay rate due to the Schwinger mechanism - creation of electron-positron pairs in strong electric fields [52].

\section{Related theories}

We conclude this section with comments on the relation of the effective Lagrangian approach to earlier theoretical frameworks.

\section{Berry's phase theory of polarization:}

In the Berry's phase theory of polarization 47, 29, 48, 49, 44, the groundstate expectation value of the twist operator $e^{-i \frac{2 \pi}{L} \hat{X}}$, which shifts the phase of electron wave functions on site $j$ by $-\frac{2 \pi}{L} j$ [44], plays a crucial role. It was revealed that the real part of a quantity

$$
w=\frac{-i}{2 \pi} \ln \left\langle 0\left|e^{-i \frac{2 \pi}{L} \hat{X}}\right| 0\right\rangle
$$

gives the linear-response electric polarization, $P_{\mathrm{el}}=-\mathrm{Re} w$ [48, while its imaginary part gives a criterion for metal-insulator transition, i.e., $D=4 \pi \operatorname{Im} w$ is finite in insulators and divergent in metals [49. The present effective action is regarded as a non-adiabatic (finite electric field) extension of $w$. To give a more accurate argument, recall that the effective Lagrangian can be expressed as

$$
\mathcal{L}(F) \sim \frac{-i \hbar}{\tau L} \ln \left(\left\langle 0\left|e^{-\frac{i}{\hbar} \tau(H+F \hat{X})}\right| 0\right\rangle e^{\frac{i}{\hbar} \tau E_{0}}\right)
$$


for $d=1$. Let us set $\tau=h / L F$ and consider the small $F$ limit. For insulators we can replace $H$ with the groundstate energy $E_{0}$ to have $\mathcal{L}(F) \sim w F$ in the linear-response regime. Thus the real part of Heisenberg-Euler's expression 20. for the non-linear polarization $P_{\mathrm{HE}}(F)=-\partial \mathcal{L}(F) / \partial F$ naturally reduces to the Berry's phase formula $P_{\mathrm{el}}$ in the $F \rightarrow 0$ limit (cf. eq.(27) below). Its imaginary part, which is related to the decay rate as $\operatorname{Im} P_{\mathrm{HE}}(F)=-\frac{\hbar}{2} \frac{\partial \Gamma(F) / L}{\partial F}$, reduces to $-D / 4 \pi$ and gives the criterion for the transition, originally proposed for the zero field case.

\section{Non-Hermitian quantum mechanics}

The dielectric breakdown of Mott insulators was also studied in the framework of non-Hermitian quantum mechanics [17, 67. Fukui and Kawakami studied a non-Hermitian Hubbard model in which the leftward and rightward hopping integral are assumed to be unequal [17. The non-Hermiticity is assumed to represent the coupling of the system with a "dissipative environment". With the Bethe ansatz solution they have observed the gap between the groundstate and the first excited state to close when the hopping asymmetry is large enough. It seems that the remaining question is to relate this result with measurable quantities.

\subsection{Zener breakdown of band insulators revisited -}

\section{Non-adiabatic geometric phase and the Schwinger mechanism}

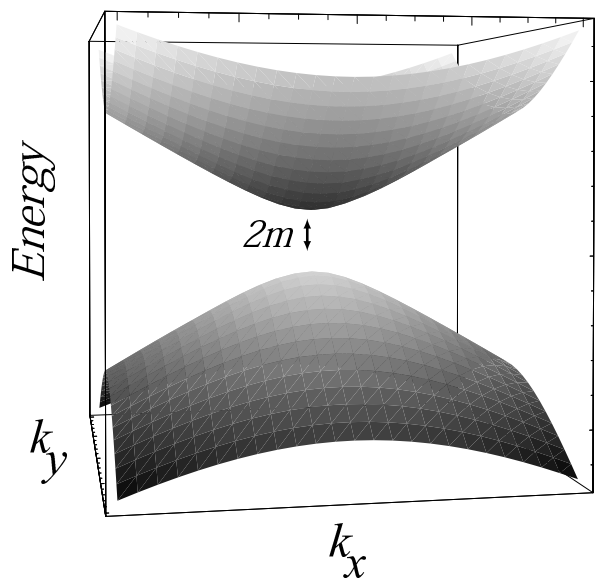

(a)

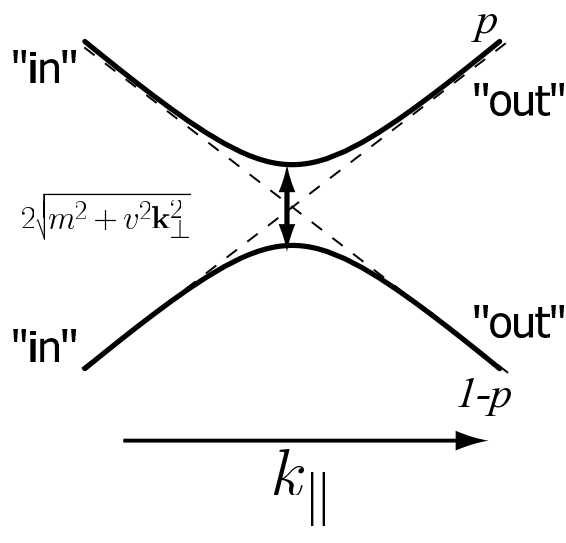

(b)

Fig. 9. (a) Energy levels of a Dirac band in 2D. (b) A one dimensional slice of the higher dimensional Dirac band in which carriers (doublons and holes) are created by an external electric field in the $k_{\|}$direction. 
Before examining the dielectric breakdown of correlated electron systems, let us first discuss the dielectric breakdown of band insulators in an electric field $\mathbf{F}$ within the effective-mass picture. This will turn out to be heuristic, since we can obtain an analytic expression for the effective Lagrangian which can be readily applied to general band insulators.

For simplicity we take a pair of hyperbolic bands $\varepsilon_{ \pm}(\mathbf{k})= \pm \sqrt{V^{2}+v^{2} k^{2}}$ (considered here in $d$ spatial dimensions), where $2 V$ is the band gap, $-(+)$ denote the valence (conduction) band, and $v$ the asymptotic slope of the dispersion.

We first obtain the groundstate-to-groundstate transition amplitude with the time-dependent gauge in the periodic boundary condition. There, a timedependent AB-flux in units of the flux quantum, $\phi(\tau)=F L \tau / h$ (with the electronic charge $e=1$ and $L$ being the system size), is introduced to induce an electric field $F$, which makes the Hamiltonian time dependent as

$$
H(\phi(\tau))=\sum_{\mathbf{k}, \alpha= \pm} \varepsilon_{\alpha}\left(\mathbf{k}+\frac{2 \pi}{L} \phi(\tau) \mathbf{e}_{\|}\right) c_{\alpha}^{\dagger}(\mathbf{k}) c_{\alpha}(\mathbf{k}) .
$$

Here $\mathbf{e}_{\|}$is the unit vector parallel to $\mathbf{F}$, and $c_{\alpha}^{\dagger}(\mathbf{k})$ the creation operator with spin indices dropped. If we denote the ground state of $H(\phi)$ as $|0 ; \phi\rangle$ and its energy as $E_{0}(\phi)$, the groundstate-to-groundstate transition amplitude reads

$$
\Xi(\tau)=\left\langle 0 ; \phi(\tau)\left|\hat{T} e^{-\frac{i}{\hbar} \int_{0}^{\tau} H(\phi(s)) d s}\right| 0 ; \phi(0)\right\rangle e^{\frac{i}{\hbar} \int_{0}^{\tau} E_{0}(\phi(s)) d s},
$$

where $\hat{T}$ stands for the time ordering. The effective Lagrangian $\mathcal{L}(F)$ for the quantum dynamics is defined from the asymptotic behavior, $\Xi(\tau) \sim$

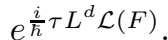

The dynamics of the one-body model can be solved analytically (Fig 9 (b)), since we can cut the dispersion in $d$ spatial dimensions into slices, each of which reduces to Landau-Zener's two band model in 1D 38, 68. Namely, if we decompose the $k$ vector as $\left(\mathbf{k}_{\perp}, k_{\|}\right)$, where $\mathbf{k}_{\perp}\left(k_{\|}\right)$is the component perpendicular (parallel) to $\mathbf{F}$, where each slice for a given $\mathbf{k}_{\perp}$ is a copy of Landau-Zener's model with a gap $\Delta_{\text {band }}(\mathbf{k}) \equiv 2 \sqrt{V^{2}+v^{2} k_{\perp}^{2}}$. The LandauZener transition takes place around the level anti-crossing for which $k_{\|}+$ $\frac{2 \pi}{L} \phi(\tau)$ moves across the Brillouin zone(BZ) in a time interval $\delta \tau=h / F$. The process can be expressed as a scattering and the Bogolubov coefficients between the "in" and "out" states (see Fig 9) is given by the solution to the two band problem, i.e.,

$$
\begin{aligned}
& c_{+}^{\dagger}(\mathbf{k}) \rightarrow \sqrt{1-p(\mathbf{k})} e^{-i \chi(\mathbf{k})} c_{+}^{\dagger}(\mathbf{k})+\sqrt{p(\mathbf{k})} c_{-}^{\dagger}(\mathbf{k}), \\
& c_{-}^{\dagger}(\mathbf{k}) \rightarrow-\sqrt{p(\mathbf{k})} c_{+}^{\dagger}(\mathbf{k})+\sqrt{1-p(\mathbf{k})} e^{i \chi(\mathbf{k})} c_{-}^{\dagger}(\mathbf{k}) .
\end{aligned}
$$

Here the tunneling probability for each $\mathbf{k}$ is given by the Landau-Zener(LZ) formula 38,68 ,

$$
p(\mathbf{k})=\exp \left[-\pi \frac{\left(\Delta_{\text {band }}(\mathbf{k}) / 2\right)^{2}}{v F}\right] .
$$


On the other hand, the phase $\chi(\mathbf{k})=-\theta(\mathbf{k})+\gamma(\mathbf{k})$ appearing in the Bogolubov coefficients consists of the trivial dynamical phase, $\hbar \theta(\mathbf{k})=\int_{0}^{\delta \tau} \varepsilon_{+}(\mathbf{k}+$ $\left.\frac{2 \pi}{L} \phi(s) \mathbf{e}_{\|}\right) d s$, and the Stokes phase [68, 25],

$$
\gamma(\mathbf{k})=\frac{1}{2} \operatorname{Im} \int_{0}^{\infty} d s \frac{e^{-i\left(\Delta_{\text {band }}(\mathbf{k}) / 2\right)^{2} s}}{s}\left[\cot (v F s)-\frac{1}{v F s}\right] .
$$

The Stokes phase, a non-adiabatic extension of Berry's geometric phase [10, depends not only on the topology of the path but, unlike the adiabatic counterpart, also on the field strength $F[26$. In terms of the fermion operators the groundstate is obtained by filling the lower band $|0 ; \phi\rangle=\prod_{\mathbf{k}} c_{-}^{\dagger}(\mathbf{k}-$ $\left.\frac{2 \pi}{L} \phi \mathbf{e}_{\|}\right)|\mathrm{vac}\rangle$, where $|\mathrm{vac}\rangle$ is the fermion vacuum with $c_{ \pm}(\mathbf{k})|\mathrm{vac}\rangle=0$. If we assume that excited charges are absorbed by electrodes we obtain from eqs.(23), (24)

$$
\begin{aligned}
& \operatorname{Re} \mathcal{L}(F)=-F \int_{\mathrm{BZ}} \frac{d \mathbf{k}}{(2 \pi)^{d}} \frac{\gamma(\mathbf{k})}{2 \pi}, \\
& \operatorname{Im} \mathcal{L}(F)=-F \int_{\mathrm{BZ}} \frac{d \mathbf{k}}{(2 \pi)^{d}} \frac{1}{4 \pi} \ln [1-p(\mathbf{k})],
\end{aligned}
$$

where the dynamical phase $\theta$ cancels the factor $e^{\frac{i}{\hbar} \int_{0}^{\tau} E_{0}(\phi(s)) d s}$ in eq. (23).

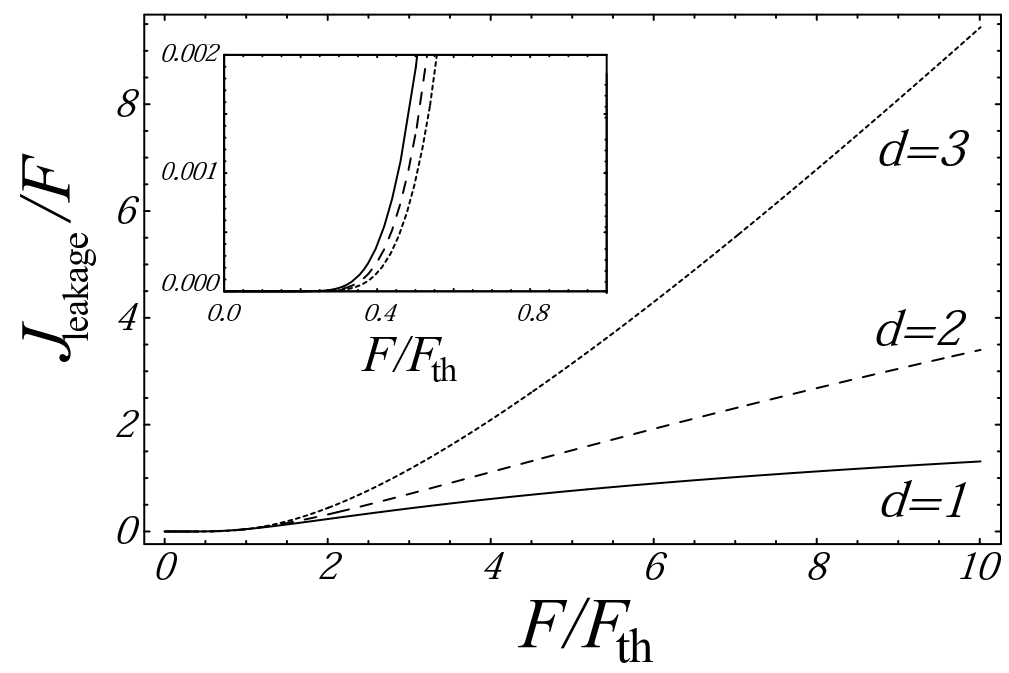

Fig. 10. The dependence of the conductivity on the electric field in the non-linear regime for band insulators with spatial dimension $d=1,2,3$. The inset zooms in the threshold region.

Integration over $\mathbf{k}$ in eq. (27) leads to the groundstate decay rate per volume for a $d$-dimensional hyperbolic band, 


$$
\begin{aligned}
\Gamma(F) / L^{d}= & \frac{F}{(2 \pi)^{d-1} h}\left(\frac{F}{v}\right)^{(d-1) / 2} \\
& \times \sum_{n=1}^{\infty} \frac{1}{n^{(d+1) / 2}} e^{-\pi n \frac{V^{2}}{v F}}\left[\operatorname{erf}\left(\sqrt{\frac{n v \pi^{3}}{F}}\right)\right]^{d-1} .
\end{aligned}
$$

The threshold for the tunneling is governed by the most nonlinear (actually essentially singular) factor in the above formula, namely $e^{-\pi n \frac{V^{2}}{v F}}$, so that the threshold electric field is given by

$$
F_{\mathrm{th}}=\frac{V^{2}}{v} .
$$

Although an analytic integration eq. (27) is possible for a Dirac band (= hyperbolic valence and conduction bands), the expression is valid for general band dispersions. In fig. 10, the leakage current divided by the field strength, which is proportional to $\Gamma(F) / F L^{d}$, is plotted for the spatial dimension $d=1,2,3$. The $F$-dependence is essentially

$$
J_{\text {leakage }} \propto F^{(d+1) / 2} e^{-\pi \frac{F_{\text {th }}}{F}},
$$

which has a threshold behavior as shown in the inset of the figure. Above the threshold, two regimes exist. In the medium field regime, the current scales as $j_{\text {leakage }} \sim F^{(d+1) / 2}$ where the power depends on $d$. However, when the field strength is even stronger, the error function appearing in eq.(28), which is due to the lattice structure (with the $\mathbf{k}$ integral restricted to the BZ), starts to take an asymptote $(\operatorname{erf}(x) \sim(2 / \sqrt{\pi}) x)$. Then various factors (including a power of $F$ ) cancel with each other, and the leakage current in the $F \rightarrow \infty$ limit approaches a universal function,

$$
J_{\text {leakage }} \propto \Gamma(F) / L^{d} \rightarrow-\frac{F}{h} \ln \left[1-\exp \left(-\pi \frac{F_{\text {th }}}{F}\right)\right],
$$

where the $d$ dependence disappears up to a trivial $d$-dependent numerical factor. This prediction on the non-linear transport can be checked experimentally including low-dimensional systems such as carbon nanotubes $(d=1)$. Graphene $(d=2)$ is also interesting, but this system has a massless Dirac dispersion, so that a special treatment should be required.

\section{Comparison to Heisenberg-Euler-Schwinger's results in non-linear QED}

Let us have a closer look at the decay of the QHE vacuum. In 1936, Heisenberg and Euler studied Dirac particles in strong electric fields, and discussed non-linear optical responses of the QED vacuum — vacuum polarization in terms of an effective Lagrangian [20. Later, Schwinger refined their approach and calculated the vacuum decay rate [52]1. Up to the one-loop level,

1 For references on the effective-action approach of non-linear electrodynamics, see 14, 15. 
Schwinger calculated the vacuum-to-vacuum transition amplitude using the proper time regularization method to obtain

$$
\Delta \mathcal{L}^{\mathrm{QED}}(F)=\frac{1}{8 \pi^{2}} \int_{0}^{\infty} \frac{d s}{s^{2}}\left[F \cot (F s)-\frac{1}{s}\right] e^{-i s m_{e}^{2}}
$$

for (3+1)-dimensional QED, where $m_{e}$ is the electron mass. The integrand has a pole in the complex domain and has an imaginary part, which gives

$$
\Gamma(F)^{\mathrm{QED}} / L^{d}=\frac{\alpha F^{2}}{2 \pi^{2}} \sum_{n=1}^{\infty} \frac{1}{n^{2}} \exp \left(-\frac{n \pi m_{e}^{2}}{|F|}\right),
$$

the famous Schwinger's formula for the electron-positron pair creation rate [52], where $\alpha=1 / 137$ is the fine-structure constant.

Thus the expression for the QED effective Lagrangian, eq.(32), coincides with the Stokes phase for the non-adiabatic Landau-Zener tunneling, except for a difference in the momentum integral. As we have mentioned above, an important difference in lattice systems is that the momentum integral is limited to the Brilliouin zone, and the decay-rate acquires an extra factor (compare eq.(28) with erf with eq.(33) ). This modification changes the strong field limit of the leakage current which leads to the universal expression (eq.(31)). Another important difference, which is quantitative, appears in the threshold voltage: The threshold for band insulators $E_{\mathrm{th}}^{\mathrm{band}}=F_{\mathrm{th}}^{\mathrm{band}} / e=V^{2} /$ vae ( $a$ : lattice constant) is many orders smaller than the threshold for the QED instability $E^{\mathrm{QED}}=\frac{m_{e}^{2} c^{3}}{\hbar} \sim 10^{16} \mathrm{~V} / \mathrm{cm}$. For example, if we have an insulator with parameters $a=10^{-7} \mathrm{~cm}, v=2 t_{\text {hop }}=1 \mathrm{eV}, V=1 \mathrm{eV}$, then the threshold becomes as small as $E_{\mathrm{th}}^{\text {band }}=10^{7} \mathrm{~V} / \mathrm{cm}$.

Heisenberg and Euler's original aim was to discuss non-linear optical properties of the vacuum in terms of $\Delta \mathcal{L}$. In fact, they calculated the effective Lagrangian in the presence of both electric and magnetic fields [20, and obtained

$$
\Delta \mathcal{L}^{\mathrm{QED}}(F)=C \frac{\mathbf{E}^{2}-\mathbf{B}^{2}}{2}+\frac{2 \alpha^{2}}{45 m_{e}^{4}}\left[\left(\mathbf{E}^{2}-\mathbf{B}^{2}\right)^{2}+7(\mathbf{E} \cdot \mathbf{B})^{2}\right]+\ldots,
$$

where $C$ is a diverging constant that we drop after renormalization. The electric polarization can be obtained from the real part of the effective action via

$$
\Delta P(F)=\frac{\partial}{\partial F} \Delta \mathcal{L}(F) .
$$

If we plug in eq(34), the non-linear polarization of Dirac particles becomes

$$
\begin{aligned}
\Delta P & =\frac{2 \alpha^{2}}{45 m_{e}^{4}}\left(-4 B^{2} E+14 B_{\|}^{2} E+4 E^{3}\right)+\ldots, \\
& =\sum_{n=1}^{\infty} P^{(n)}(\mathbf{B}) E^{n}
\end{aligned}
$$


where $B_{\|}$is the component of $\mathbf{B}$ parallel to $\mathbf{E}$, and $P^{(n)}(\mathbf{B})$ the $n$-th order non-linear polarization. Thus we can examine nonlinear polarizations and cross correlations (a combined effect of $\mathbf{E}, \mathbf{B}$ ) with the effective Lagrangian, as touched upon in Table 1

2.4 Dielectric breakdown in a Mott insulator - many-body Landau-Zener transition and a nonequilibrium phase diagram
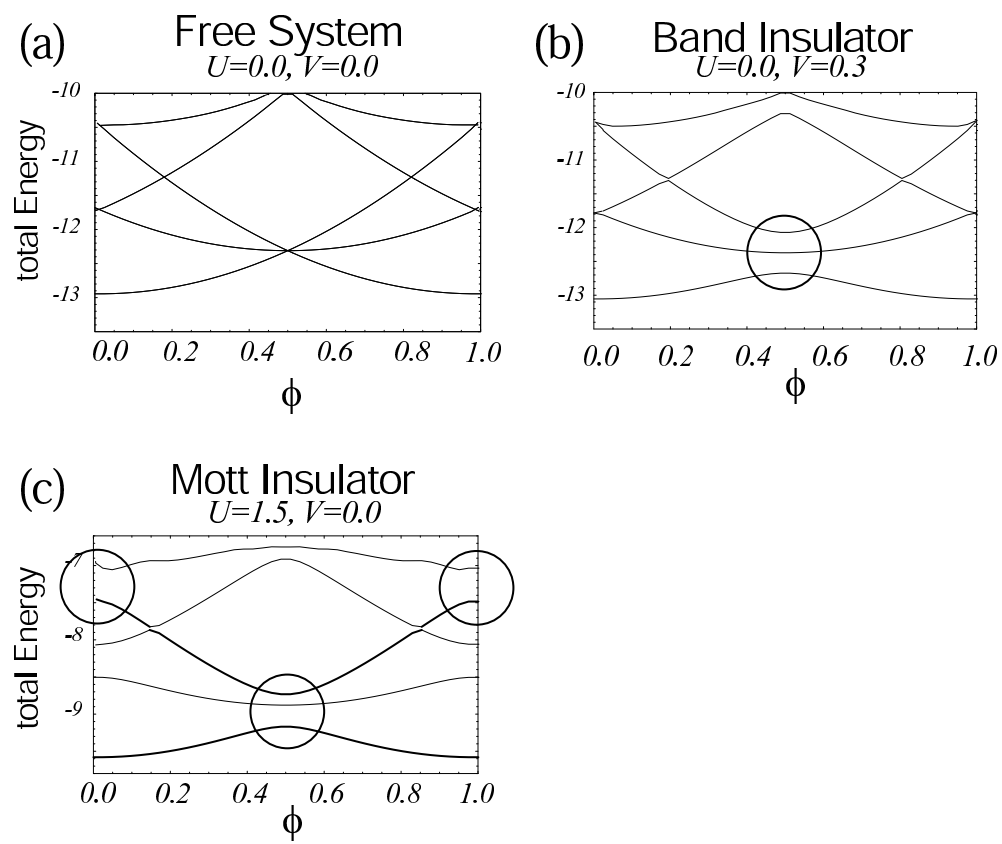

Fig. 11. Adiabatic spectrum $E_{n}(\phi)$ for a finite system ( $L=10$ here) obtained by the Lanczos method. We plot low-lying excitations in the half-filled subspace $N_{\uparrow}=N_{\downarrow}=L / 2$. (a) a noninteracting system in a free space, (b) a band insulator $\left(U / t_{\text {hop }}=0, V / t_{\text {hop }}=0.3\right)$, and (c) a Mott insulator $\left(U / t_{\text {hop }}=1.5, V / t_{\text {hop }}=0\right)$. The circles indicate avoided level crossings.

Before applying the effective Lagrangian approach to the dielectric breakdown of Mott insulators, we need to examine the excitation spectra, which is displayed in fig 11. There we plot, for the half-filling, the many-body energy levels of the Hamiltonian,

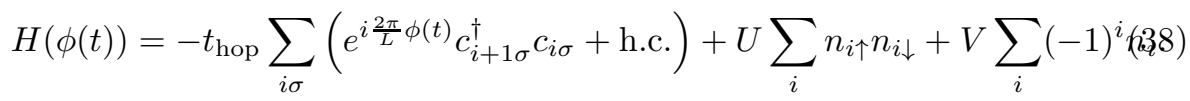

Here $U$ is the Hubbard repulsion, $V$ a staggered potential to introduce valence and conduction bands, so that $U=V=0$ corresponds to a noninteracting 
system in a free space, $U=0, V \neq 0$ a band insulator, and a large $U$ and $V=0$ a Mott insulator. In the figure, we have only plotted charge excitations (where the charge rapidities are excited in the language of Bethe-ansatz solution). As can been seen, levels cross in the free model while in the band and the Mott insulators an energy gap separates the ground state from excited states. The gap is $2 V$ for the band insulator. The Hubbard Hamiltonian eq.(38) with $U \neq 0, V=0$ is also exactly solvable in $1 \mathrm{D}$. Woynarovich used the Bethe ansatz method [65, 66] to study the ground state as well as the excited states (see also [56, 37, 36]). The two solid lines in Mott insulator's spectrum correspond to the ground state and the charge-excited state with one pair of complex charge rapidities, quantum numbers appearing in the Bethe-ansatz solution. The energy gap $\Delta E(U)$ between these states are known to converge to the Mott gap $\Delta_{\text {Mott }}(U)$ in the limit of infinite system,

$$
\Delta E(U) \rightarrow \Delta_{\mathrm{c}}(U)
$$

An important feature in the spectrum of the Mott insulator is that level repulsion occurs at many places over the excited states. The repulsion comes from Umklapp electron-electron scattering, i.e., a scattering process in which the momentum sum changes by reciprocal lattice vectors. In band insulators level repulsions obviously come from one-body scattering as we have seen above.

Why have we first looked at the adiabatic spectrum? There is an important relation between the adiabatic energy and the current expectation value. From the Hellmann-Feynman theorem, i.e., $\frac{d E}{d \lambda}=\frac{\langle\Psi|\partial H / \partial \lambda| \Psi\rangle}{\langle\Psi \mid \Psi\rangle}$ for $H(\lambda)|\Psi(\lambda)\rangle=$ $E(\lambda)|\Psi(\lambda)\rangle$, we obtain

$$
\begin{aligned}
J_{n}(\phi) & =\langle n ; \phi|J(\phi)| n ; \phi\rangle \\
& =\left(\frac{L}{2 \pi}\right) \frac{\partial E_{n}(\phi)}{\partial \phi}
\end{aligned}
$$

which is valid for all $\phi$. If we expand it around $\phi=0$, we get

$$
J_{n}(\phi)=J_{n}(0)+\left(\frac{L}{2 \pi}\right) \frac{\partial^{2} E_{n}(0)}{\partial \phi^{2}} \phi+O\left(\phi^{2}\right) .
$$

Using $\phi=F L t / h$ and defining the transport coefficients $\mathcal{D}_{n}$ by $J_{n}(\phi)=$ $J_{n}(0)+\mathcal{D}_{n} F t+O\left(F^{2}\right)$, we obtain

$$
\mathcal{D}_{n}(L)=\left(\frac{L}{2 \pi}\right)^{2} \frac{\partial^{2} E_{n}(0)}{\partial \phi^{2}} .
$$

When we focus on a dissipationless adiabatic transport at $T=0$, the total current thus reads

$$
\langle J(t)\rangle=\mathcal{D}_{0}(L) F t,
$$


which is determined by the Drude weight (charge stiffness) $\mathcal{D}_{0}(L)$. As we can see in Fig 11, even for insulators $((\mathrm{b})$ and $(\mathrm{c}))$, the Drude weight $\mathcal{D}_{0}(L)$ of a finite system is not necessarily zero. If we remember Kohn's criterion [31] for metal-insulator transitions, stated as

$$
\lim _{L \rightarrow \infty} \mathcal{D}_{0}(L)=\left\{\begin{array}{cl}
0 & \text { insulator } \\
\text { finite } & \text { perfect metal, }
\end{array}\right.
$$

we can see that we must go to the limit of infinite systems to distinguish metals from insulators. Indeed, the problem of taking the infinite-size limit is also occurs in the study of dielectric breakdown in Mott insulators as we shall see later.

\section{Short-time behavior — an exact diagonalization result}

Since the time evolution of many-body systems cannot be treated analytically, we employ numerical methods to time-integrate in two steps - for short-time behavior and long-time behavior. For the short-time evolution in dielectric breakdown of Mott insulators we exactly diagonalize the timedependent Schrödinger equation as follows: First we start from the ground state of $H(\phi=0)$ at time $t=0$. The wave function evolves with the phase that increases as

$$
\phi(t)=0 \rightarrow F L t / h .
$$

Here $F=e a E$ is the field strength, $L$ the length of the chain. We numerically solve the time-dependent Schrödinger equation,

$$
i \frac{d}{d t}|\Psi(t)\rangle=H(\phi(t))|\Psi(t)\rangle
$$

We choose the initial state to be the ground-state $|0\rangle$ of $H(0)$, which is obtained here by the Lanczos method. The time integration of the state vector, which, being a many-body state, has a huge dimension, requires a reliable algorithm. So we adopt here the Cranck-Nicholson method that guarantees the unitary time evolution, where the time evolution is put into a form,

$$
|\Psi(t+\Delta t)\rangle=e^{-i \int_{t}^{t+\Delta t} H(t)} d t|\Psi(t)\rangle \simeq \frac{1-i \Delta t / 2 H(t+\Delta t / 2)}{1+i \Delta t / 2 H(t+\Delta t / 2)}|\Psi(t)\rangle,
$$

which is unitary by definition. Here the time step is taken to be small enough $\left(d t=1.0 \times 10^{-2}\right.$ with the time in units of $\hbar / t$ hereafter $)$ to ensure convergence for $L \leq 10$, for which the dimension of the Hamiltonian is $\sim 10^{4}$. We have concentrated on the total $S^{z}=0$ subspace with $N_{\uparrow}=N_{\downarrow}=L / 2$.

\section{Evolution of the total current}

We first plot in Fig 12(b) the result for the expectation value of the current density averaged over the sites, $J=-\frac{i t}{L} \sum_{i, \sigma}\left(e^{i \frac{2 \pi}{L} \phi(t)} c_{i+1 \sigma}^{\dagger} c_{i \sigma}-\right.$ h.c. $)$. The 


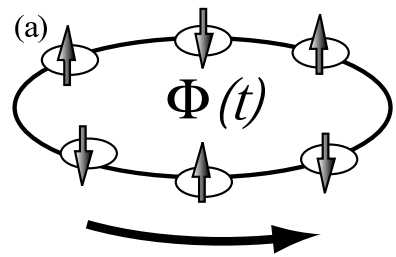

\section{Electric Field $F$} current $J(t)$
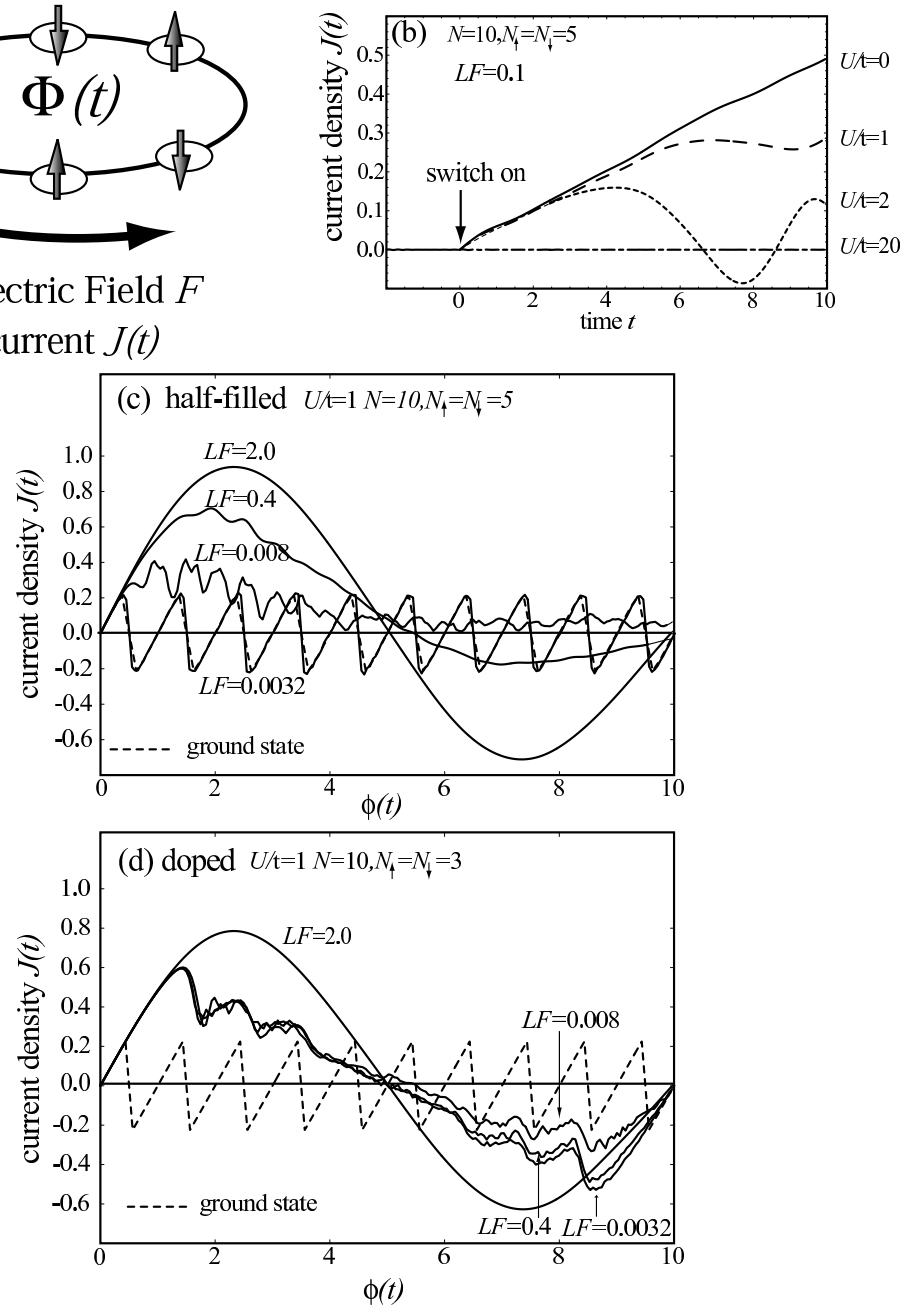

Fig. 12. (a) The sample geometry, where an $\mathrm{AB}$ flux, $\phi(t)=L F t$, increasing linearly with time induces an electric force through Faraday's law. (b) Time evolution of the current, $J(t)$, for a half-filled, 10-site Hubbard model for various strengths of the Hubbard repulsion, $0 \leq U / W \leq 5$ ( $W=4 t_{\text {hop }}$ is the non-interacting band width) for a fixed electric field $F=1 / 10 L$. Time is measured in units of $\tau_{t} \equiv \hbar / t_{\text {hop }}$, $L F$ in $t_{\text {hop }}$, and $J(t)$ in $1 / \tau_{t}$. The range of the time in this panel is restricted to a range of the AB-flux $0 \leq \phi \leq 1$. (c) A wider plot of the current for various values of $F$ with a fixed $U / W=0.25$, again for the half-filled case. Here the horizontal axis is $\phi$. (d) A plot similar to (c) for a non-half-filled case $\left(N_{\uparrow}=N_{\downarrow}=3<L / 2=5\right)$.

behavior of $J(t)$ for a fixed value of the electric field $F$ is seen to fall upon three regimes when $U$ is varied: A perfect metallic behavior $(J(t) \propto t)$ when the electrons are free $(U / W=0)$, an insulating behavior $(J(t)=0)$ when 
the interaction is strong enough $(U / W \gg 1)$, and an intermediate regime of $U / W$ where $J$ is finite with some oscillations for finite systems. In contrast, a non-half-filled system in nonequilibrium $(F \neq 0)$ has a time evolution that is distinct from the ground-state behavior (Fig 12(d)). The difference has its root in the spectral property as will be discussed later.

If we look at the behavior over several periods $(0<\phi<10)$ for a fixed value of $U / W$ for the half-filled (Fig[12(c)) and for a non-half-filled case (Fig[12(d)), the result may be summarized as follows:

(i) Small $F$ regime (Mott insulator preserved at half filling)

A drastic difference between the half-filled and doped systems appears for small $F$. When half-filled, $J(t)$ in the limit of $F \rightarrow 0$ smoothly approaches a periodic saw-tooth behavior with periodicity $\phi=1$, which is the ABoscillation of the ground-state current.

(ii) Moderate $F$ regime (metal)

In this regime, the current in the half-filled case is non-zero and shows oscillatory behaviors (seen typically in data for $L F=0.008$ in Fig $12(\mathrm{c})$ ).

(iii) Large $F$ regime (perfect metal)

When the electric field $F$ becomes large enough, the system behaves as a kind of metal. The current $J(t)$ exhibits a long-period $\left(\Delta \Phi=\Phi_{0} L\right)$ oscillation, which is the Bloch oscillation, a hallmark of a metal.

The averaged current,

$$
\langle J\rangle=\frac{1}{T} \int_{0}^{T}\langle J(t)\rangle d t,
$$

integrated over a quarter of the Bloch period (with $\phi(T)=\frac{L}{4}$ ) is plotted against $F$ in Fig 13 for various values of $U$. We can see that $\langle J\rangle$ becomes nonzero rather abruptly at the metallization as $F$ is increased, where the threshold electric field increases and the $F$ - dependence becomes weaker when we increase $U / t$. Just after the metallization some oscillation (in the $F$ - dependence this time) is seen for finite systems.

Evolution of the survival probability

In order to calculate the decay rate introduced above, we compute the temporal evolution of the ground-state survival probability,

$$
P_{0}(t)=\left|\left\langle 0 ; \phi(s)\left|\hat{T} e^{-\frac{i}{\hbar} \int_{0}^{t} H(\phi(s)) d s}\right| 0 ; 0\right\rangle\right|^{2},
$$

where $|0 ; \phi\rangle$ denotes the ground-state of $H(\phi)$. The survival probability is related to the decay rate of the ground-state by $P_{0}(t)=e^{-\Gamma t}$.

The short-time feature in the survival probability is expected to be described by the single Landau-Zener transition between the ground-state and the lowest excited state, displayed by the two bold lines in the figure 2 , that

\footnotetext{
${ }^{2}$ In Fig[14 (a), three states appear in the circle. However, transition from the ground-state to the middle state is forbidden by symmetry.
} 


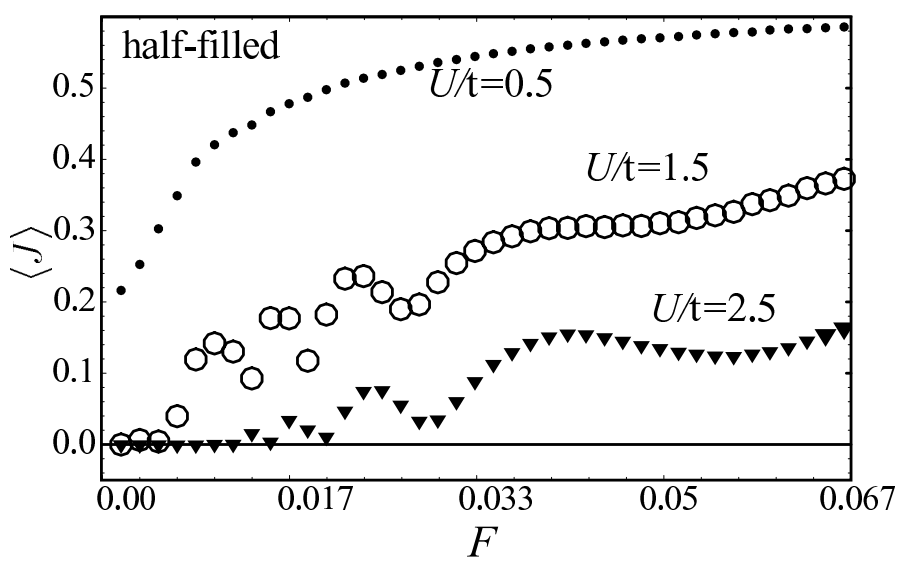

Fig. 13. Dependence of the averaged current $\langle J(t)\rangle$ on $F$ for various values of $U / t$ for the half-filled Hubbard model with $L=6$.
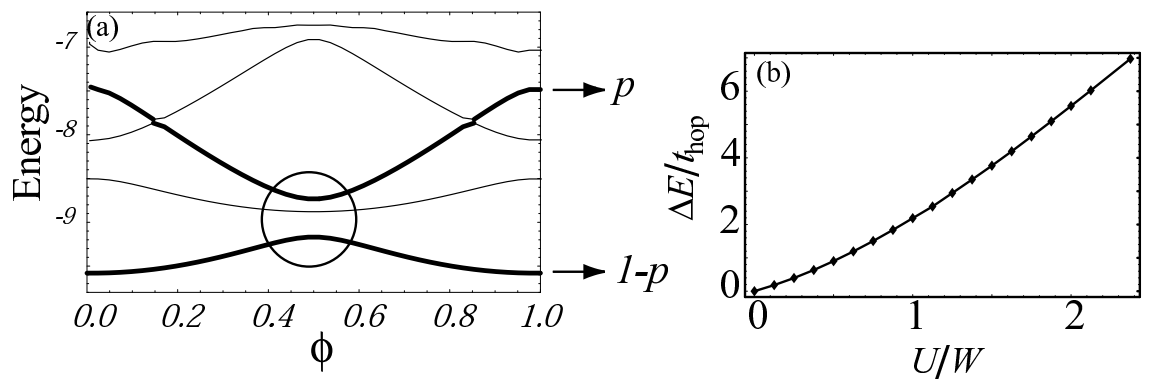

Fig. 14. (a) Spectrum of the half-filled Hubbard model $H(\phi)$ for $0 \leq \phi \leq 1$. Bold lines represent the ground state and the first state among the tunneling-allowed excited states, respectively. (b) $U / W$-dependence of the Mott gap $\Delta E$, encircled in (a). $W=4 t_{\text {hop }}$ is the non-interacting band width, and system size is $L=10$ and $U / t_{\mathrm{hop}}=1.5$.

takes place around $\phi=\frac{1}{2}$ (Fig 14(a)). If we concentrate on the two levels, the time evolution operator at $t=\Delta t\left(\Delta t=\frac{h}{F L}\right.$ is defined as the time when $\phi(\Delta t)=1$ is reached) is approximated by a $2 \times 2$ matrix,

$$
U_{2 \text { level }}(t=\Delta t)=\left(\begin{array}{cc}
\sqrt{1-p} e^{-i \chi} & -\sqrt{p} \\
\sqrt{p} & \sqrt{1-p} e^{i \chi}
\end{array}\right),
$$

where the tunneling probability $p$ is given by the Landau-Zener formula [38, 68, 55,

$$
p=\exp \left(-\pi \frac{F_{\mathrm{th}}^{\mathrm{LZ}}}{F}\right), \quad F_{\mathrm{th}}^{\mathrm{LZ}}=\frac{\left[\Delta_{c}(U) / 2\right]^{2}}{v} .
$$

Here, $\Delta_{c}(U)$ is the excitation gap (Fig 14(b)), $v=2 t_{\text {hop }}$, and $\chi$ the sum of dynamical and Stokes phases. 
In order to verify eq.(51), we can numerically calculate the survival probability $P_{0}(t)$ from $t=0$ to $t=\Delta t$ for various $U$ and $F$ (Fig 15). After determining the tunneling probability from $p=1-P_{0}(\Delta t)$, we plot it against the diabaticity parameter $\frac{\left(\Delta_{c}(U) / 2\right)^{2}}{v F}$. The data points(Fig[15(b)) for various values of $U$ fall around a common line, which is just the prediction of the Landau-Zener formula. The agreement is better for smaller values of $U$ where we can treat the Umklapp term as a perturbation.

\section{Long-time behavior — a time-dependent DMRG result}

The conclusion obtained in the previous section with the exact diagonalization is that the short-time behavior after the electric field is switched on is dominated by the single Landau-Zener transition between the ground state and the first excited state. However, several important questions remain, e.g.,

Will the first transition remain finite in the infinite-size limit? Indeed, Kohn's criterion (44) asserts that the $\phi$ dependence of the ground-state energy of a Mott insulator should vanish for $L \rightarrow \infty$. This implies that the adiabatic flow (Fig. 11(c)) should become flat in this limit, which may seem to indicate that the transition will be washed out. However, this contradicts with the expression for the threshold $F_{\text {Zener }}=\frac{\left[\Delta_{c}(U) / 2\right]^{2}}{v}($ eq. (1511) $)$, which remains finite in the $L \rightarrow \infty$ limit. Since this expression is obtained in a small system and in the small $U$ limit, there is a possibility that this breaks down. Surprisingly, we shall show that this expression survives in large systems even when $U$ is not small (Fig. 18).

The effect of pair annihilation After the first transition, we expect the system to undergo further transitions to higher-energy levels. This process, however, should be couterbalanced by another processes, the pair annihilation of doublons and holes. These processes, which do not conserve the total momentum in general, are caused by the Umklapp scattering. Thus the pair creation (= Landau-Zener transitions to high-energy states) tends to be offset by pair annihilation, which implies that the decay rate of the ground state may become smaller compared to the single Landau-Zener transition cas 3 .

These questions have motivated us to study the dielectric breakdown in the half-filled Hubbard model for longer time periods, which is accomplished by the time-dependent density matrix renormalization group method. A version of the real-time DMRG was first intruduced by Cazalilla and Marston with a truncated DMRG Hilbert space and a renormalized Hamiltonian 12. Precision of their method degrades rapidly in the long-time limit, since an update of the Hilbert space is lacking. Recently, Vidal proposed an improved method for simulating time-dependent phenomena in one-dimensional lattice systems

3 A similar problem has been studied from a general point of view by Wilkinson and Morgan 64. 

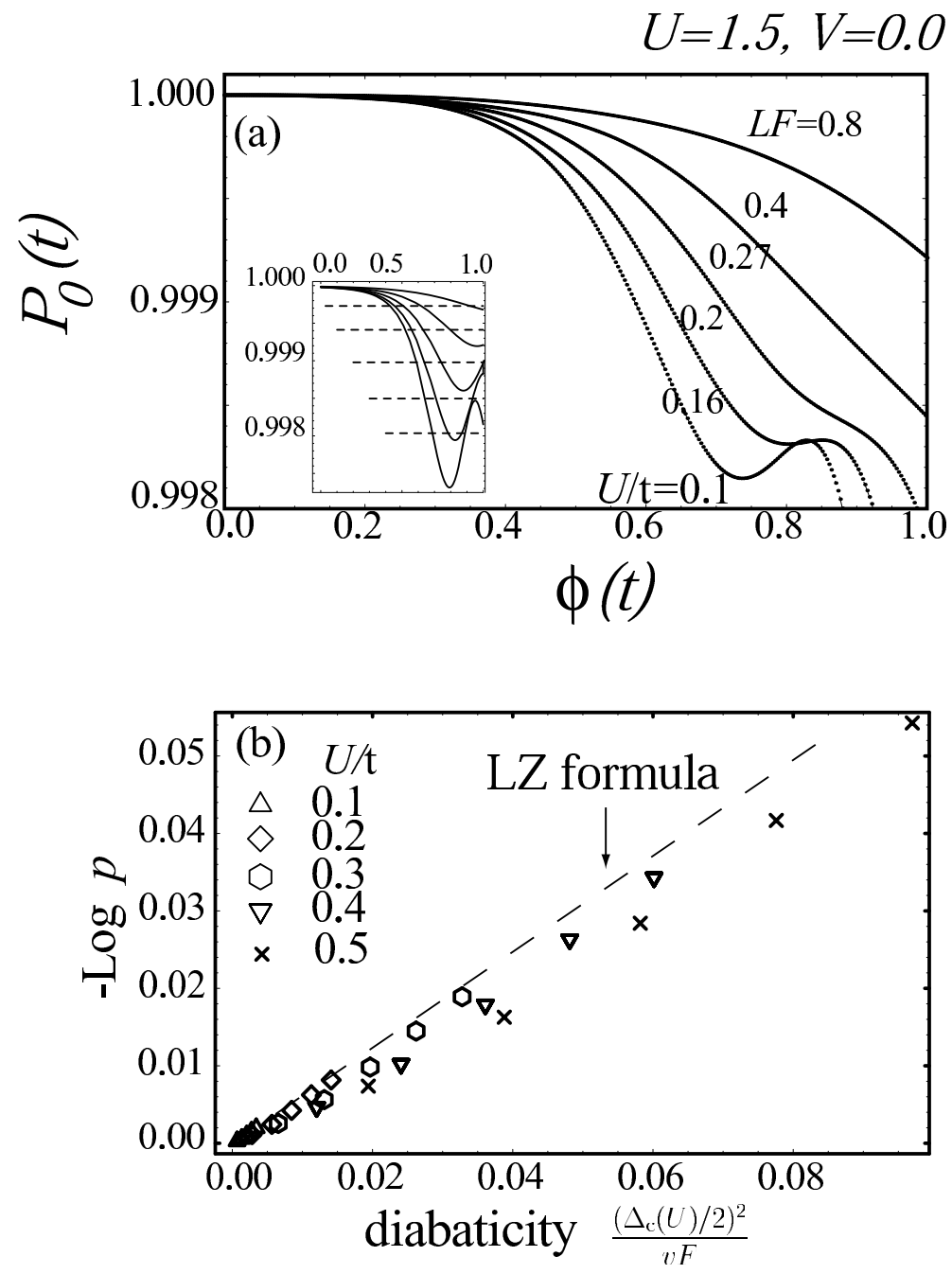

Fig. 15. (a) Short-time temporal evolution of the survival probability $P_{0}(t)$ in the half-filled Hubbard model $\left(L=10, N_{\uparrow}=N_{\downarrow}=5\right)$ for various values of $F$ with $U / t=0.1$. The inset shows the solutions of the LZS equation with its asymptotic values indicated as dashed horizontal lines. (b)The transition probability $p$, with $P(t=\Delta t)=1-p$, plotted against the diabaticity parameter. The dashed line is the prediction of the Landau-Zener formula.

employing the Trotter-Suzuki decomposition 61, 62. White and Feiguin [63. as well as other groups [18] modified this idea and combined it with the finitesize DMRG algorithm.

If we denote the DMRG wave function as 


$$
|\Psi\rangle=\sum_{l \alpha_{j} \alpha_{j+1} r} \psi_{l \alpha_{j} \alpha_{j+1} r}|l\rangle\left|\alpha_{j}\right\rangle\left|\alpha_{j+1}\right\rangle|r\rangle,
$$

where $|l\rangle,|r\rangle$ is the basis of the truncated Hilbert space with dimension $m$ and $\left|\alpha_{j}\right\rangle,\left|\alpha_{j+1}\right\rangle$ are the two sites that bridge the left and right blocks in the DMRG procedure. By employing the Trotter-Suzuki decomposition,

$$
e^{-i d t H} \simeq e^{-i d t H_{1} / 2} e^{-i d t H_{2} / 2} \ldots e^{-i d t H_{2} / 2} e^{-i d t H_{1} / 2},
$$

we can apply the time-evolution operator $e^{-i d t H_{j} / 2}$ to the $j$-th wave function as

$$
\left(e^{-i d t H_{j} / 2} \psi\right)_{l \alpha_{j} \alpha_{j+1} r}=\sum_{\alpha_{j}^{\prime} \alpha_{j+1}^{\prime}}\left(e^{-i d t H_{j} / 2}\right)_{\alpha_{j} \alpha_{j+1} ; \alpha_{j}^{\prime} \alpha_{j+1}^{\prime}} \psi_{l \alpha_{j}^{\prime} \alpha_{j+1}^{\prime} r} .
$$

After applying $e^{-i d t H_{j} / 2}$, we diagonalize the density matrix and move to the next link just as in the usual finite-size algorithm. One cycle of this procedure results in an evolution of time by $d t$, and we can repeat it as many times as we wish. Compared with the version by Cazalilla-Marston [12, this algorism has higher precision and we can simulate non-equilibrium excited states efficiently [18], although one drawback of the t-dependent DMRG is that we can only treat systems with open boundary conditions.

Here we study transient behaviors of the one-dimensional Hubbard model with open boundary condition. We use the time-independent gauge, for which the Hamiltonian is

$$
H(F)=-t_{\text {hop }} \sum_{j, \sigma}\left(c_{j+1 \sigma}^{\dagger} c_{j \sigma}+\text { h.c. }\right)+U \sum_{j} n_{j \uparrow} n_{j \downarrow}+F \hat{X},
$$

where $\hat{X}=\sum_{j} j n_{j}$ is the position operator representing the tilted potential. As in the previous section, we start the time evolution from the $F=0$ groundstate $|0\rangle$ obtained by the usual finite-size DMRG. The wave function in this gauge is simply

$$
|\Psi(t)\rangle=e^{-i t H(F)}|0\rangle
$$

which is obtained with the t-dependent DMRG.

\section{Evolution of the charge density}

We first discuss the temporal evolution of the charge density, $n_{j}(t)=$ $\left\langle\Psi(t)\left|n_{j}\right| \Psi(t)\right\rangle$, after the electric field is switched on at $t=0$. At half-filling the initial distribution is $n_{j}(t)=1$. After the application of the electric field, a charge density wave (CDW) pattern is formed when the electric field is not too strong (Fig. 16(a)). This state is stationary and the density profile do not change any further. The pattern is formed because the boundary condition breaks the translational symmetry, where the amplitude of the pattern corresponds to the polarization $\Delta P(F)$ induced by the field. When the electric field 
(a) $F=0.1$

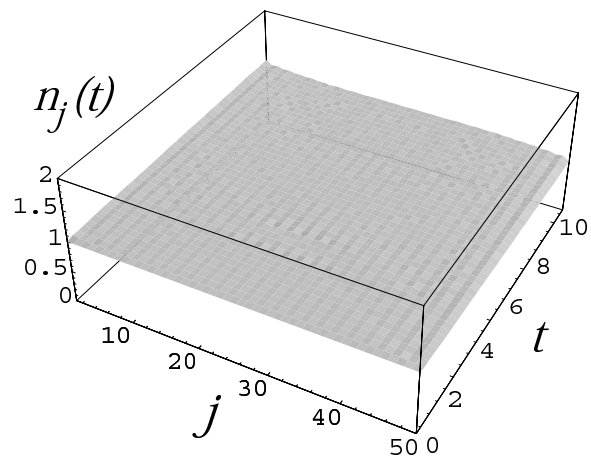

(c) $F=0.1$

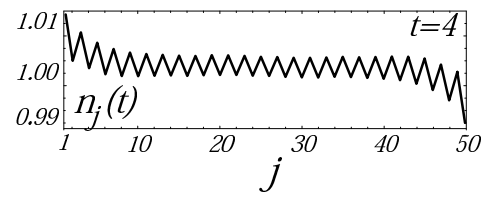

(b) $F=1.0$

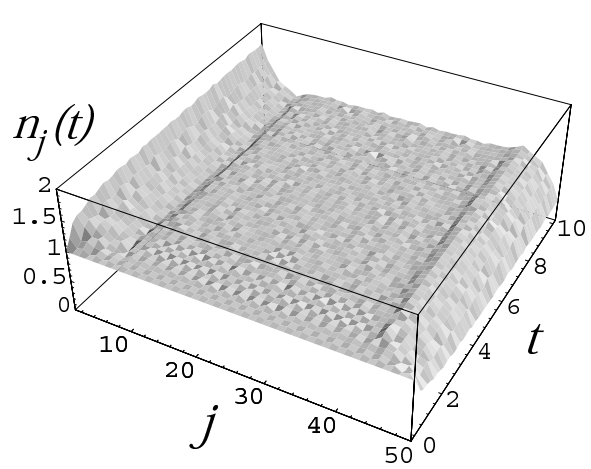

Fig. 16. Temporal evolution of the charge density $n_{j}(t)$ in the half-filled Hubbard model with $m=150, L=50, d t=0.02$ for $F=0.1$ (a) and $F=1.0$ (b). (c) depicts a cross section of (a) for $t=4$.

becomes stronger, charge transfers start to occur, with charge accumulation and charge depletion being formed around the edges in an open-boundary chain (Fig 16). This is a sign that the ground state collapses due to quantum tunneling.

The decay rate of the ground state

The groundstate-to-groundstate transition amplitude is, in the time-independent gauge,

$$
\Xi(t)=\left\langle 0\left|e^{-\frac{i}{\hbar} \tau(H+F \hat{X})}\right| 0\right\rangle e^{\frac{i}{\hbar} t E_{0}},
$$

where we denote the ground state of $H$ as $|0\rangle$ and its energy as $E_{0}$. Figure 17 (a) shows the temporal evolution of the ground-state survival probability $|\Xi(t)|^{2}$ for a system with $U / t_{\text {hop }}=3.5$. As time evolves, the slope of $-\ln |\Xi(t)|^{2}(\propto$ the decay rate) decreases after an initial stage, which implies a suppression of the tunneling from the short-time behavior. This should indicate that charge excitations are initially produced due to the Landau-Zener tunneling from the ground state to the first excited states, but that scattering among the excited states become important as the population of the excitations grows. In other words, pair annihilation of carriers becomes important and acts to suppress 

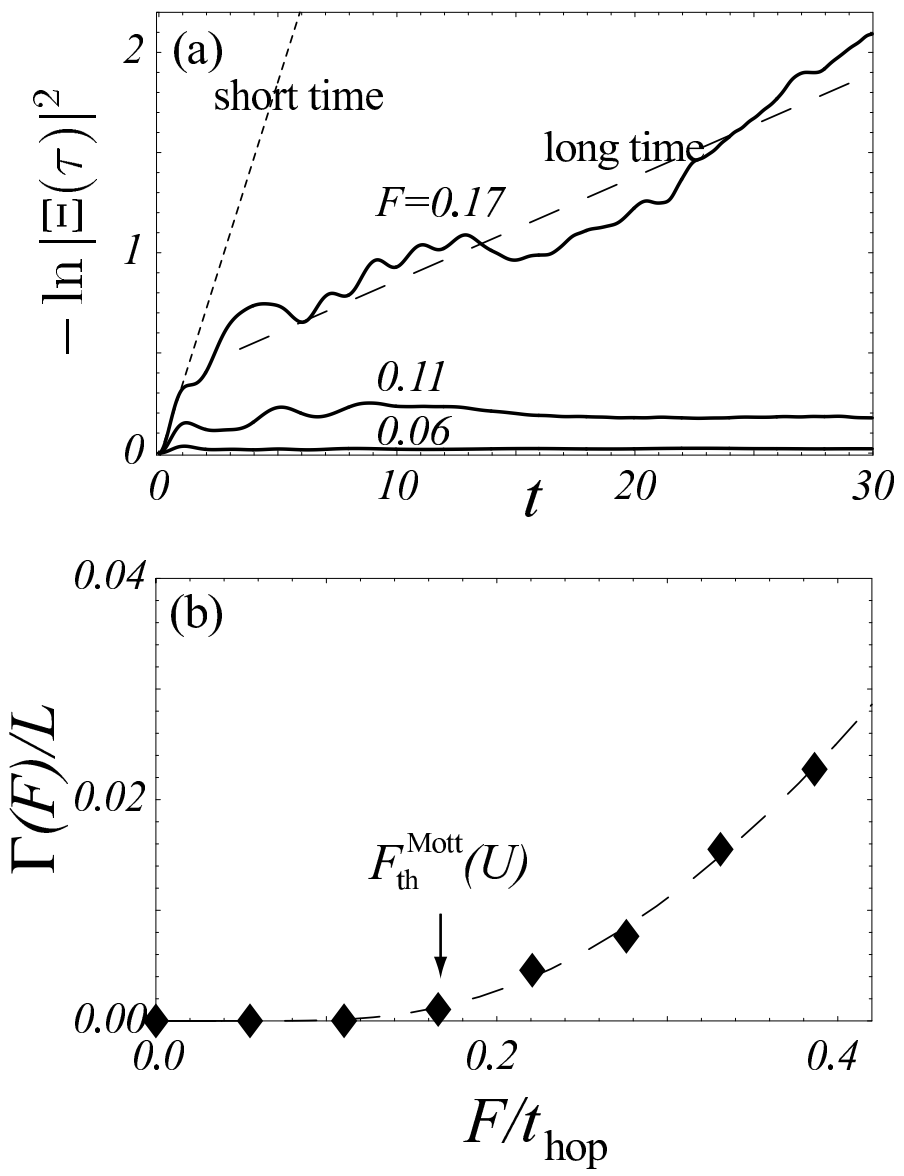

Fig. 17. (a) Temporal evolution of the ground-state survival probability $|\Xi(t)|^{2}$ after the electric field $F$ is switched on at $t=0$ in the 1D half-filled Hubbard model with $U / t_{\text {hop }}=3.5$, obtained with the time-dependent DMRG for $L=50$ with the size of the DMRG Hilbert space $m=150$ and the time step $d t=0.02$. The dashed line represents $-\ln |\Xi(t)|^{2}=\Gamma(F) t+c$ for $F / t_{\text {hop }}=0.17$, while the dotted line delineates the initial slope (the short-time behavior). (b) The decay rate versus $F$ in the half-filled Hubbard model. Dashed curve is a fit to eq.(58), where $F_{\mathrm{th}}^{\mathrm{Mott}}(U)$ is the threshold.

the tunneling rate. We have determined $\Gamma(F)$ from the long-time behavior with a fitting $-\ln |\Xi(t)|^{2}=\Gamma(F) t+$ const.

The decay rate per length $\Gamma(F) / L$ is plotted in Fig[17(b), where we have varied the system size $(L=30,50)$ to check the convergence. $\Gamma(F) / L$ is seen to remain vanishingly small until the field strength exceeds a threshold. To characterize the threshold $F_{\mathrm{th}}(U)$ for the breakdown we can evoke the form obtained above for the one-body system. The formula (eq. (28) for $d=1$ 
with the error function ignored and the factor of 2 recovered for the spin degeneracy),

$$
\Gamma(F) / L=-\frac{2 F}{h} a(U) \ln \left[1-\exp \left(-\pi \frac{F_{\mathrm{th}}^{\mathrm{Mott}}(U)}{F}\right)\right],
$$

is originally derived for one-body problem, and an obvious interest here is whether the formula can be applicable if we replace the one-body $F_{\mathrm{th}}^{\mathrm{band}}$ with the many-body $F_{\mathrm{th}}^{\mathrm{Mott}}(U)$. In the above we have added a factor $a(U)$, a parameter representing the suppression of the quantum tunneling. The dashed line in Fig 17 (b) is the fitting to the formula for $U / t_{\text {hop }}=3.5$, where we can see that the fitting, including the essentially singular form in $F$, is surprisingly good, given a small number of fitting parameters. The value of $a(U)$ turns out to be close to but smaller than unity (taking between 0.77 to 0.55 as $U / t$ is increased from 2.5 to 5.0 ).

If we perform this for various values of $U$ we can construct a "nonequilibrium (dielectric-breakdown) phase diagram", as displayed in Fig 18, which

plots the $U$ dependence of $F_{\mathrm{th}}^{\mathrm{Mott}}$. The dashed line is the prediction of the Landau-Zener formula [58,

$$
F_{\mathrm{th}}^{\mathrm{LZ}}(U)=\frac{\left[\Delta_{\mathrm{c}}(U) / 2\right]^{2}}{v}
$$

For the size of the Mott (charge) gap we use the Bethe-ansatz result, 16.

$$
\Delta_{\mathrm{c}}(U)=\frac{8 t_{\mathrm{hop}}}{U} \int_{1}^{\infty} \frac{\sqrt{y^{2}-1}}{\sinh \left(2 \pi y t_{\mathrm{hop}} / U\right)} d y,
$$

with $v / t_{\text {hop }}=2$. As can be seen, the DMRG result and the Landau-Zener result agrees surprisingly well.

\subsection{Long-time behavior and a mapping to a quantum random walk}

Since many levels should be involved in the above pair creation/annihilation processes, next thing we want to have is a statistical mechanical setup for the time evolution of the Mott insulator. The problem at hand is a closed quantum system in external driving forces (e.g., electric fields), which are represented by a time varying parameter $\phi(t)$ of the Hamiltonian. We want to discuss the asymptotic solution of the time-dependent Schrödinger equation

$$
i \hbar \frac{d}{d t}|\Psi(t)\rangle=H(\phi(t))|\Psi(t)\rangle .
$$

We introduce $|n ; \phi\rangle$ as the set of eigenstates of the time-dependent Hamiltonian $H(\phi)$, and denote the energy eigenvalue as $E_{n}(\phi)$, i.e., $H(\phi)|n ; \phi\rangle=$ $E_{n}(\phi)|n ; \phi\rangle(\operatorname{Fig}[19)$. Since $|n ; \phi\rangle$ forms a complete orthonormal basis, the wave function $|\Psi(t)\rangle$ can be expanded as 


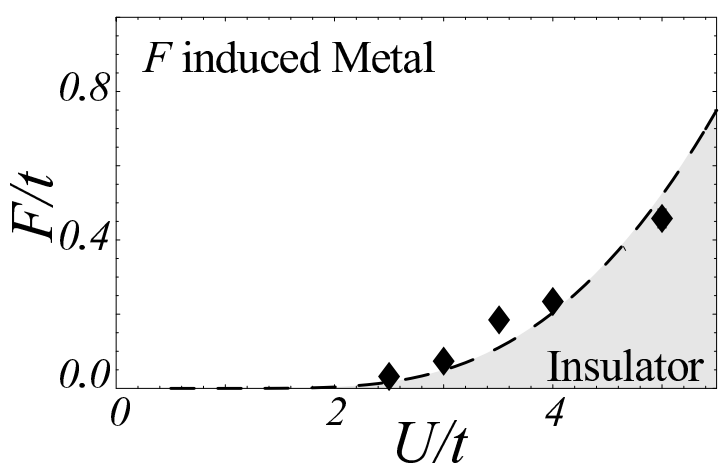

Fig. 18. The dielectric-breakdown phase diagram on the $(U, F)$ plane for the onedimensional Hubbard model. The symbols are the threshold $F_{\mathrm{th}}^{\mathrm{Mott}}(U)$ obtained by fitting the decay rate $\Gamma(F) / L$ to eq.(58)), while the dashed line is the prediction $F=F_{\mathrm{th}}^{\mathrm{LZ}}(U)$ of the Landau-Zener formula eq.(59).

$$
|\Psi(t)\rangle=\sum_{n} \psi(n, t) e^{-\frac{i}{\hbar} \int_{0}^{t} E_{n}(s) d s}|n ; \phi(t)\rangle
$$

with coefficients $\psi(n, t)=\left\langle n ; \phi(t)\left|\hat{T} e^{-i \int_{0}^{t} H(\phi(s)) d s}\right| 0 ; 0\right\rangle / e^{-\frac{i}{\hbar} \int_{0}^{t} E_{n}(s) d s}$. Note that we have removed the contribution from the dynamical phase $\int_{0}^{t} E_{n}(s) d s / \hbar$ in the definition of $\psi(n, t)$. Although the evolution depends on the detail of the system $(H(\phi))$, we can deduce some universal features that depend only on the feature of the energy levels, i.e., distribution of level repulsion in the spectrum.

Each energy level is subject to the Landau-Zener tunneling to neighboring levels in a time period $\Delta t / 2$, and is most conveniently expressed in terms of the transfer matrix representation 7 , 43. To this end, we denote the pairs as

$$
\Psi(n, \tau)=\left(\begin{array}{c}
\psi_{L}(n, \tau) \\
\left.\psi_{R}(n, \tau)\right)
\end{array}\right)
$$

and the time evolution "rule" can be expressed as

$$
\Psi(n, \tau+1)=P_{n+1} \Psi(n+1, \tau)+Q_{n-1} \Psi(n-1, \tau),
$$

where $P_{n}\left(Q_{n}\right)$ is the upper (lower) half of a $2 \times 2$ unitary matrix,

$$
U_{n}=\left(\begin{array}{cc}
a_{n} & b_{n} \\
c_{n} & d_{n}
\end{array}\right), \quad P_{n}=\left(\begin{array}{cc}
a_{n} & b_{n} \\
0 & 0
\end{array}\right), \quad Q_{n}=\left(\begin{array}{cc}
0 & 0 \\
c_{n} & d_{n}
\end{array}\right) .
$$

The diagonal elements of $U_{n}$ represent the Landau-Zener transition from the $n$-th level to $(n-1)$ or $(n+1)$-th level, where the explicit form is

$$
U_{n}=\left(\begin{array}{cc}
\sqrt{p_{n}} e^{i \beta_{n}} & \sqrt{1-p_{n}} e^{i \gamma_{n}} \\
-\sqrt{1-p_{n}} e^{-i \gamma_{n}} & \sqrt{p_{n}} e^{-i \beta_{n}}
\end{array}\right)
$$


(a) Half-filled (Mott insulator)

n (b) idealized spectrum
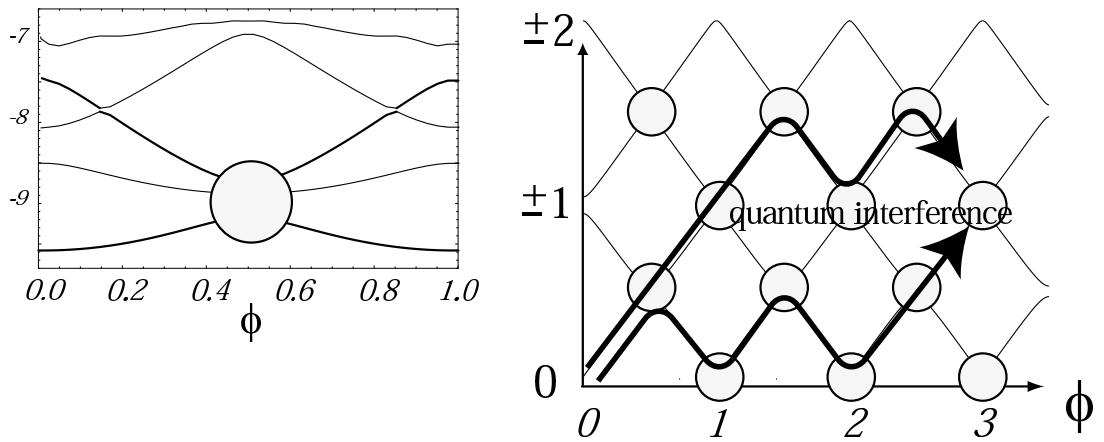

Fig. 19. (a) The spectrum of the half-filled Hubbard model. The circle corresponds to Landau-Zener transition between the two energy levels which can be expressed by a $2 \times 2$ unitary matrix (eq.(66) ). (b) Idealized energy levels where level anti-crossings are expressed by circles. Quantum interference takes place when contributions from different paths are considered.

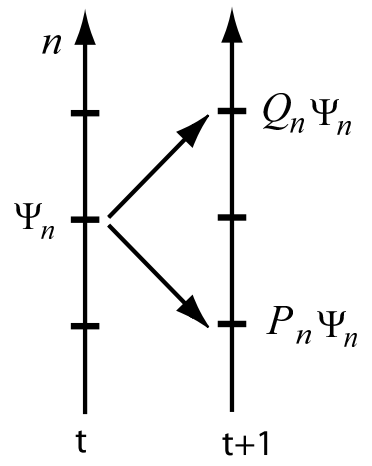

Fig. 20. Application of $Q_{n}$ and $P_{n}$ in the quantum random walk.

Here the Landau-Zener tunneling probability $p_{n}$ depends on the ratio of the Zener threshold field $F_{\text {Zener }}^{n}$ and the electric field $F$ as

$$
p_{n}=\exp \left(-\pi \frac{F_{\mathrm{Zener}}^{n}}{F}\right)
$$

where $F_{\text {Zener }}^{n}$ generically depends on $n$.

If we regard $\Psi(n, \tau)=\left(\begin{array}{c}\psi_{L}(n, \tau) \\ \psi_{R}(n, \tau)\end{array}\right)$ as a "qubit" on "site" $n$, eq. (64) defines an evolution of a one-dimensional quantum walk with a reflecting boundary at $n=0$ corresponding to the ground-state $(\mathrm{Fig}[19(\mathrm{c}))$. A quantum walk is a quantum counterpart of the classical random walk. Models with essentially equivalent ideas have appeared in various fields: to name a few, quantum transport and dissipation [11, 58, quantum Hall effect [13], optics [51, 5] and 
recently in quantum information [45, 53, 35, 33, 54, 42, 34, 22, 30, 40, 28, 40, 6. In the field of quantum information (see e.g. (46)), introduced by Aharonov, Ambainis, Kempe and Vazirani in 2001 [27, the quantum walk is arousing interest in hope of revealing new features in the quantum algorithms (for reviews see [28, 40, 6]). Researches stem into many directions, e.g., the effect of absorbing boundary conditions [22, 30, 53], higher-dimensional systems [54, 40, 32, localization in systems with internal degrees of freedom 21, and many powerful analytical techniques are being developed.

An important feature of the quantum walk, as opposed to the classical walk, is that different transition paths interfere with each other quantum mechanically. We in fact find that the quantum interference leads to a $d y$ namical localization, an analog of Anderson's localization taking place in the energy space rather than in the position space. In our previous work [7] we have employed the PRQS method, a technique to treat quantum walks, to perform the path integral, and obtained the exact asymptotic distributions of the wave function for a simplified model. The resultant states can be categorized in three types depending on the strength of the electric field, as schematically plotted in Fig. 21] (A) is an adiabatic evolution that takes place in weak driving forces (electric fields). The dominant part of the distribution $\rho(n, t)$ against energy is a delta function localized around the ground-state. When the driving force become stronger, quantum tunneling broadens the delta function, as plotted in (B). The shape of the peak is maintained by a balance between tunneling and dynamical localization. (C) is the case where the driving force overwhelms the effect of dynamical localization, and the system is driven rapidly into the excited states. This, in our view, corresponds to the dielectric breakdown. 
(a) adiabatic

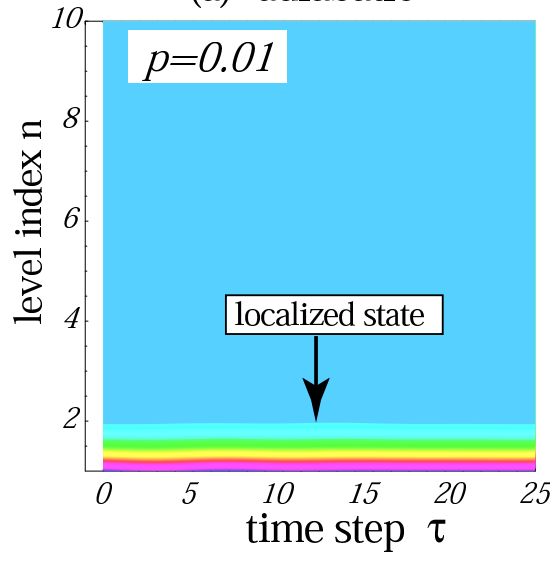

(c) delocalized

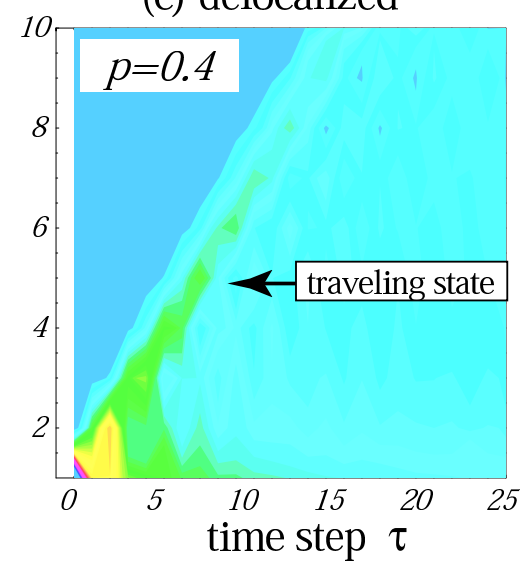

(b) localized

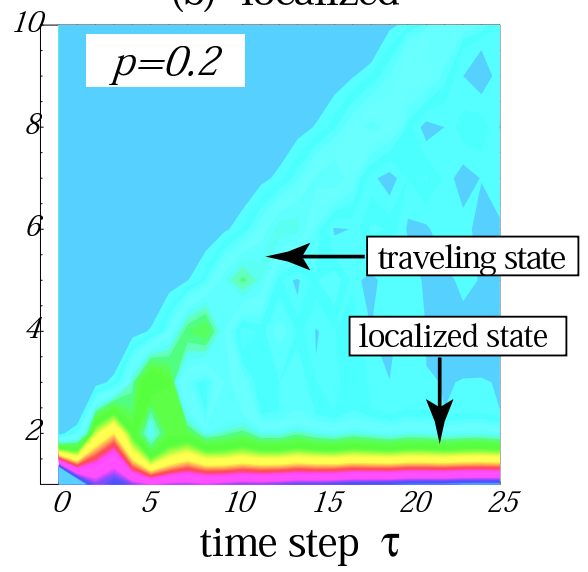

Fig. 21. The time evolution of the distributions of wave function amplitude $\rho(n, t)=|\psi(n, t)|^{2}$ in energy space. The vertical axis $n$ is the index of the energy levels. (a)For small tunneling, the distribution is localized at the ground state. (b) For intermediate tunneling, a localized state remains, while the amplitude starts to bifircate into excited states of the wave function is excited. (c) When the tunneling is larger than the threshold, the localized state disappears.

\subsection{Experimental implications}

Now we discuss experimental implication of the many-body Landau-Zener transition mechanism. In fact, there are several mechanisms which may lead to breakdown of insulators. For example, Fröhlich's electric avalanche mechanism may take place, in which a small number of excited electrons act as a seed and become accelerated by the electric field until they cooperatively destroy the insulator. We can distinguish Landau-Zener transition from the avalanche 
mechanism through the temperature dependence and from interface effects by changing the size of the sample. Another important effect is the band bending near an interface of the Mott insulator and electrodes. For a thin sample, this may lead to injection of carriers, and results in the interface Mott transition 59 .

(a)

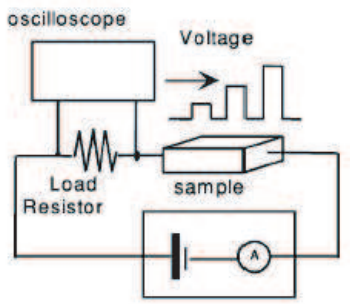

Source-measure unit

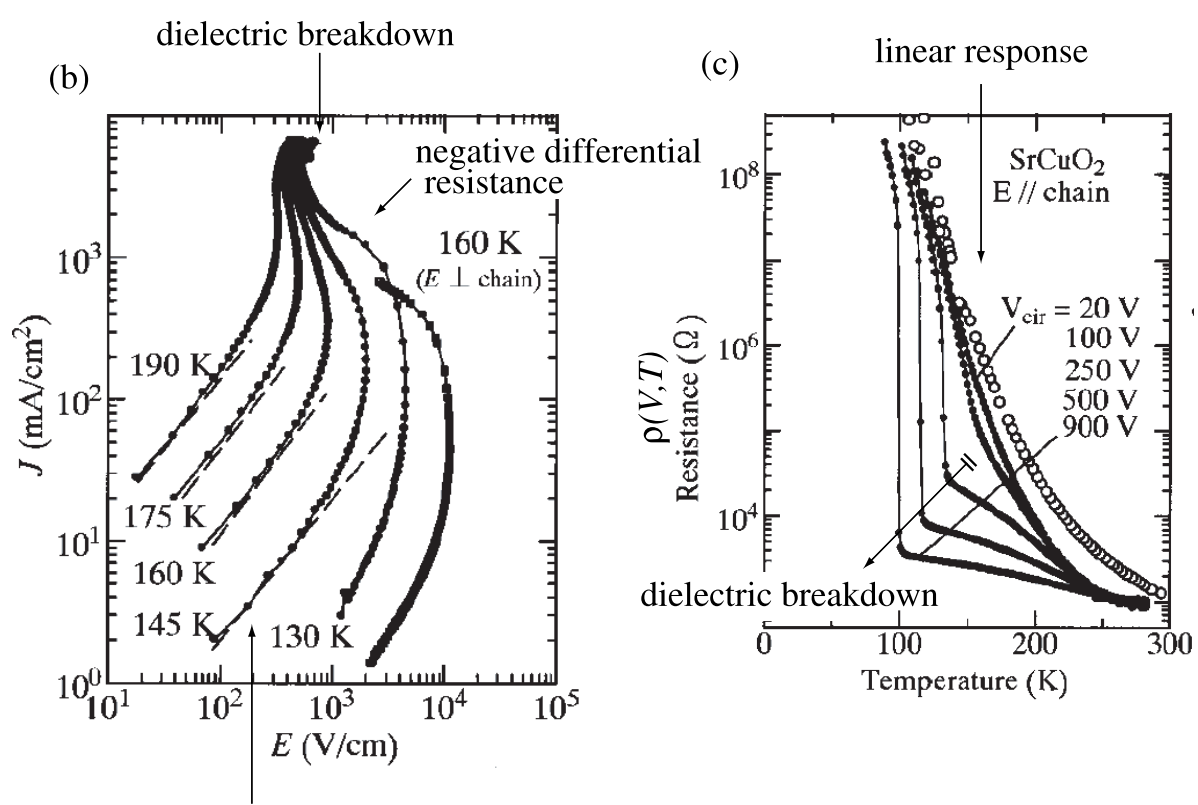

Ohmic law

(finite $T$ effect)

Fig. 22. (a) A schematic measurement circuit. (b) $J-E$ characteristics of a $\mathrm{SrCuO}_{2}$ sample. (c) Temperature dependence of the resistance for various applied voltages. After Taguchi et al. 41. 
Dielectric breakdown of one-dimensional Mott insulators was experimentally studied by Taguchi et al., who obtained the $J-E$ characteristics (Fig 22) of $\mathrm{Sr}_{2} \mathrm{CuO}_{3}$ and $\mathrm{SrCuO}_{2}$ samples 41, which are both quasi-1D, strongly correlated electron systems. Experiments were done by placing small single crystals in circuits as shown in Fig 22(a), and the voltage drop $V$ was measured while the current density $J$ was fixed. Depending on the strength of the electric field $F$, transport properties change drastically, as summarized in the following.

In weak electric fields, the $J-E$ characteristics shows an Ohmic behavior at finite temperatures. When the electric field exceeds a threshold value, the current shows a dramatic increase. Such drastic changes cannot be explained by perturbation in $F$, and we must consider non-perturbative effects, i.e., a behavior essentially singular in $F$ like $J \sim$ function of $\exp \left(-F_{\text {th }} / F\right)$, which is a typical tunneling effect with threshold $F_{\mathrm{th}}$. The temperature dependence (Fig 23) of the threshold can be fit well by $F_{\mathrm{th}}(T) / F_{\mathrm{th}}(0) \sim \exp \left(-T / T_{0}\right)$. This excludes the avalanche mechanism, for which an activation type temperature dependence $\left(F_{\mathrm{th}}^{\text {avalanche }}(T) / F_{\mathrm{th}}(0) \sim \exp \left(T_{0} / T\right)\right)$ is expected.

One indication that the breakdown is indeed quantum in nature is that the threshold extrapolates to a finite value for $T \rightarrow 0$. From the extrapolation (Fig[23(a)), we obtain a threshold,

$$
F_{\text {th }}^{\exp } \sim 10^{6}-10^{7}(\mathrm{eV} / \mathrm{cm}),
$$

for $\mathrm{Sr}_{2} \mathrm{CuO}_{3}$ and $\mathrm{SrCuO}_{2}$. The Landau-Zener result (intended for $T=0$ ) of the threshold (eq.(151)) is

$$
F_{\mathrm{th}}^{\mathrm{LZ}}(U)=\frac{\left[\Delta_{\mathrm{c}}(U) / 2\right]^{2}}{v} \sim(1 \mathrm{eV})^{2} /\left(10^{-7} \mathrm{eV} / \mathrm{cm}\right) \sim 10^{6}(\mathrm{eV} / \mathrm{cm})
$$

is comparable with the experimental result.

Interestingly, the decay rate $\Gamma(U)$ we have introduced theoretically can be measured experimentally [41]. This is done by studying the transient behavior of the current after the electric field is switched on at $t=0$. At first the current density is zero, and then becomes non-zero after a certain delay time $t=\tau(F)($ Fig 23 (b), lower inset). The authors in [1] have introduced a phenomenological percolation model to relate the delay time with the production rate $P(F)$ of the conductive domains (see [1]). In this model, conductive domains are envisaged to grow in the sample, and the current density is assumed to become finite when the left and right electrodes are connected by these domains (Fig 23(c)). This leads to a relation,

$$
P(F)=-\left(F \frac{d \tau}{d F}\right)^{-1}
$$

The experimental result for the production rate in Fig 23(b) is obtained in this way.

The nature and the microscopic origin of the "conductive domain" are not clear, but if we interpret them to be domains with a high density of charge 


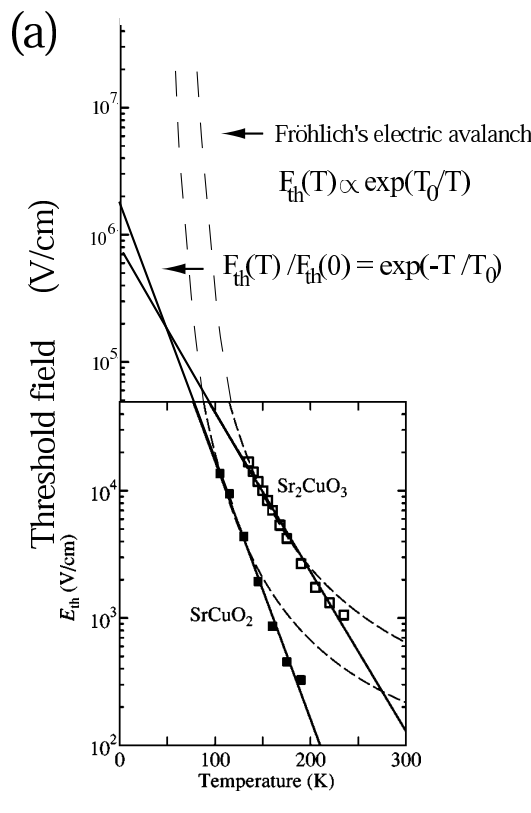

(b)

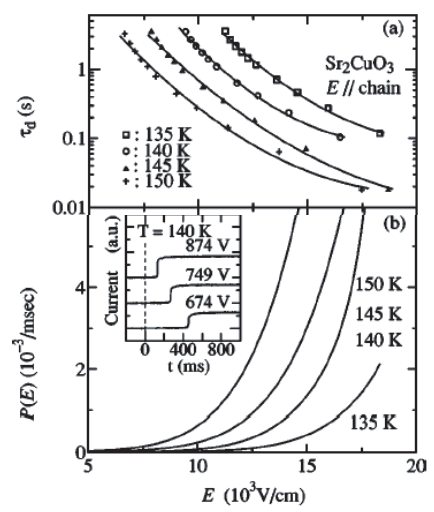

(c)

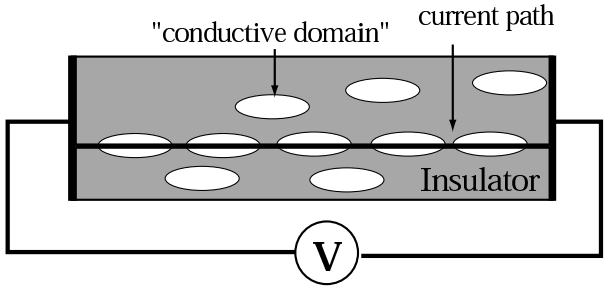

Fig. 23. An experimental result for the temperature dependence of the threshold electric field $F_{\mathrm{th}}(T)$ for the dielectric breakdown (adopted from Taguchi et al. 41]). Dashed lines correspond to a fitting to $\exp \left(T_{0} / T\right)$ predicted by Fröhlich's electron avalanche. Solid lines correspond to a fitting to $\exp \left(-T / T_{0}\right)$. (b) The electric-field dependence of the delay time $\tau_{\mathrm{d}}$ for $\mathrm{Sr}_{2} \mathrm{CuO}_{3}$. (c) The production rate of "conductive domains". The inset exemplifies the temporal evolution of the current at various applied voltages. Adopted from Taguchi et al. 41.

excitations produced by the Landau-Zener transition, the vacuum decay rate per volume $\Gamma(F) / L^{d}$ characterizes quantum tunneling from the ground-state to excited states. With the identification we expect that the decay rate and the production rate are identical, i.e.,

$$
P(F) \sim \Gamma(F) .
$$

This identification is encouraged by the field dependence of $P(F)$ (lower panel of Fig $23(\mathrm{~b}))$, which is close to the expected form $\left(\Gamma / L \sim-\frac{2 F}{h} a(U) \ln \left[1-\exp \left(-\pi \frac{F_{\mathrm{th}}^{\mathrm{Mott}}}{F}\right)\right]\right)$ of the decay rate.

In this experiment a scaling study - a systematic change of the size of the sample - was also performed to confirm that the nonlinear effect occurs in the bulk. From these observations, we conclude that the experiment by Taguchi et al. 41 can be explained by the many-body Landau-Zener tunneling mechanism. However, to be more confident, we need to know the temperature 
dependence of the threshold theoretically, which is still a challenging task in the present many-body system.

\subsection{Conclusion}

In this article, we have explained how dielectric breakdown of Mott insulators can be explained from the nonequilibrium behaviors of charge carriers, especially from their creation and annihilation processes. Both processes are the result of many-body Landau-Zener nonadiabatic tunneling transition between many-body energy levels, where charge creation processes are counterbalanced by annihilation processes. From numerical result we have obtained a nonequilibrium (dielectric-breakdown) phase diagram. If the coherence of the dynamics is preserved at sufficiently low temperatures, a quantum interference, as modeled by a quantum walk in energy space, may lead to dynamical localization, which saturates the creation process and leads to a non-equilibrium stable state. The decay rate $\Gamma(F)$ that we have discussed is a measurable quantity: it is the production rate observed by Taguchi et al. 41 in copper oxides. The experimental result is consistent with our prediction $\Gamma(F) \sim \frac{F}{2 \pi} \ln (1-p)$ when an extrapolation to zero temperature is made. It is an interesting future topic to understand the properties of the non-equilibrium stable state in more detail.

An important open question is how the energy dissipation processes take place in nonequilibrium situations. Here we have stressed that the many-body processes act effectively as a source of dissipation through scattering, but an explicit incorporation of heat-bath effects, electrode effects, etc, is left to a future problem.

Acknowledgements: We wish to thank Rytaro Arita and Norio Konno for the collaboration in the workes described here and for illuminating discussions. We also indebted to Yshai Avishai and Paul Wiegmann for illuminating discussions. TO acknowledges Masaaki Nakamura, Kazuma Nakamura, Shuichi Murakami, and Naoto Nagaosa for helpful comments.

\section{References}

1. A. Asamitsu, Y. Tomioka, H. Kuwahara, and Y. Tokura, Nature, 388:50, 1997.

2. A. Baikalov, Y. Q. Wang, B. Shen, B. Lorenz, S. Tsui, Y. Y. Sun, Y. Y. Xue, and C. W. Chu , Appl. Phys. Lett., 83:957, 2003.

3. A. Ohtomo and H. Y. Hwang, Nature, 427:423, 2004.

4. A. Sawa, T. Fujii, M. Kawasaki, and Y. Tokura, Appl. Phys. Lett., 85:4073, 2004.

5. A. Wöjcik T. Luczak P. Kurzynski A. Grudka and M. Bednarska, Phys. Rev. Lett., 93:180601, 2004.

6. A. Ambainis, International Journal of Quantum Information, 1:507, 2003.

7. T. Oka N. Konno R. Arita and H. Aoki, Phys. Rev. Lett., 94:100602, 2005.

8. T. Oka R. Arita and H. Aoki, Physica B., 759:359-361, 2005. 
9. J. E. Avron and J. Nemirovsky, Phys. Rev. Lett., 68:2212, 1992.

10. M.V. Berry, Proc.R.Soc.Lond., A 392:45, 1984.

11. G. Blatter and D.A. Browne, Phys.Rev.B, 37:3856, 1988.

12. M.A. Cazalilla and J.B. Marston, Phys. Rev. Lett., 88:256403, 2002.

13. J.T. Chalker and P.D. Coddington, J. Phys. C, 21:2665, 1988.

14. C.Itzykson and J-B. Zuber, Quantum Field Theory. McGraw-Hill, Inc., 1980.

15. W. Dittrich and H. Gies, Probing the Quantum Vacuum. Springer-Verlag, Berlin, 2000.

16. E. H. Lieb, and F. Y. Wu, Phys. Rev. Lett, 21:192, 1968.

17. T. Fukui and N. Kawakami, Phys. Rev. B, 58:1651, 1998.

18. A.J. Daley C. Kollath U. Schollwoeck G.Vidal, J. Stat. Mech.: Theor. Exp., P04005, 2004.

19. H. Oshima, K. Miyano, Y. Konishi, M. Kawasaki, and Y. Tokura, Appl. Phys. Lett., 75:1473, 1999.

20. W. Heisenberg and H. Euler, Z.Physik, 98:714, 1936.

21. N. Inui and N. Konno, Physica A, 353:133, 2005.

22. E. Bach S. Coppersmith M.P. Goldschen R. Joynt and J. Watrous, Journal of Computer and System Sciences, 69:562, 2004.

23. C. G. Callan Jr. and S. Coleman, Phys. Rev. D, 16:1762, 1977.

24. K. Inagaki, I. Terasaki, H. Mori, T. Mori, J. Phys. Soc. Jpn, 73:3364, 2004.

25. Y. Kayanuma, Phys.Rev.B, 47:9940, 1993.

26. Y. Kayanuma, Phys.Rev.A, 55:R2495, 1997.

27. D. Aharonov A. Ambainis J. Kempe and U.V. Vazirani, Proceedings of the 33rd Annual ACM Symposium on Thoery of Computing, 50, 2001.

28. J. Kempe, Contemporary Physics, 44:307, 2003.

29. R.D. King-Smith and D. Vanderbilt, Phys. Rev. B, 47:R1651, 1993.

30. T. Yamasaki H. Kobayashi and H. Imai, Phys.Rev.A, 65:032310, 2003.

31. W. Kohn, Phys. Rev. A, 133:171, 1963.

32. N. Inui Y. Konishi and N. Konno, Physical Review A:052323, 2004.

33. N. Konno, Quantum Information and Computation, 2:578, 2002.

34. N. Konno, Quantum Information Processing, 1:345, 2002.

35. N. Konno, Journal of the Mathematical Society of Japan, 57:1179, 2005.

36. R. Arita K. Kusakabe K. Kuroki and H. Aoki, J. Phys. Soc. Jpn., 66:2086, 1997.

37. K. Kusakabe and H. Aoki, J. Phys. Soc. Jpn., 65:2772, 1996.

38. L.D. Landau, Phys. Z. Sowjetunion, 2:46, 1932.

39. M. Imada, A. Fujimori, and Y. Tokura, Rev. Mod. Phys., 70:1039, 1998.

40. B. Tregenna W. Flanagan W. Maile and V. Kendon, New Journal of Physics, 5:83.1, 2003.

41. Y. Taguchi T. Matsumoto and Y. Tokura, Phys. Rev. B, 62:7015, 2000.

42. T. Namiki N. Konno and T. Soshi, Interdisciplinary Information Sciences, 10:11, 2004.

43. H. Nakamura, J.Chem.Phys., 87:4031, 1987.

44. M. Nakamura and J. Voit, Phys.Rev.B, 65:153110, 2002.

45. A. Nayak and A. Vishwanath, quant-ph/0010117, 2000.

46. M.A. Nielsen and I.L. Chuang, Quantum Computation and Quantum Information. Cambridge University Press, 2000.

47. R. Resta, Ferroelectrics, 136:51, 1992.

48. R. Resta, Phys.Rev.Lett., 80:1800, 1998.

49. R. Resta and S. Sorella, Phys.Rev.Lett., 82:370, 1999. 
50. S. Q. Liu, N. J. Wu, and A. Ignatiev, Appl. Phys. Lett., 76:2749, 2000.

51. D. Bouwmeester I. Marzoli G. P. Karman W. Schleich and J. P. Woerdman, Phys. Rev. A, 61:013410, 2000.

52. J. Schwinger, Phys. Rev., 82:664, 1951.

53. N. Konno T. Namiki T. Soshi and A. Sudbury, J. Phys. A: Math. Gen., 36:241, 2003.

54. T. D. Mackay S. D. Bartlett L. T. Stephanson and B. C. Sanders, J. Phys. A: Math. Gen., 35:2745, 2002.

55. E.C.G. Stuckelberg, Helv. Phys. Acta, 5:369, 1932.

56. B. Sutherland, Phys. Rev. Lett., 74:816, 1995.

57. T. Oka and H. Aoki, Phys. Rev. Lett., 95:137601, 2005.

58. T. Oka R. Arita and H. Aoki, Phys. Rev. Lett., 91:66406, 2003.

59. Takashi Oka and Naoto Nagaosa , Phys. Rev. Lett., 95:266403, 2005.

60. V. Ponnambalam, S. Parashar, A. R. Raju, and C. N. R. Rao, Appl. Phys. Lett., 74:206, 1999.

61. G. Vidal, Phys. Rev. Lett., 91:147902, 2003.

62. G. Vidal, Phys. Rev. Lett., 93:040502, 2004.

63. S. R. White and A. E. Feiguin, Phys. Rev. Lett., 93:076401, 2004.

64. M. Wilkinson and M. A. Morgan, Phys. Rev. A, 61:062104, 2000.

65. F. Woynarovich, J.Phys.C, 15:85, 1982.

66. F. Woynarovich, J.Phys.C, 15:97, 1982.

67. Y. Nakamura and N. Hatano, Journal of the Physical Society of Japan, 75:104001, 2006.

68. C. Zener, Proc. R. Soc. London. Ser. A, 137:696, 1932.

69. C. Zener, Proc. Roy. Soc. London, 145:523, 1934. 